\title{
RNF20 and RNF40 regulate vitamin D receptor-dependent signaling in inflammatory bowel disease
}

\author{
Robyn Laura Kosinsky ${ }^{1}{ }^{1}$ - Maria Zerche ${ }^{2}$ Ana Patricia Kutschat ${ }^{2} \cdot$ Asha Nair $^{3}$ - Zhenqing Ye ${ }^{3}$ - Dominik Saul ${ }^{4}$. \\ Maximilian von Heesen ${ }^{2}$ Jessica J. Friton ${ }^{1} \cdot$ Ana Carolina Schwarzer ${ }^{2} \cdot$ Nadia Paglilla $^{2}$ - Shehzad Z. Sheikh ${ }^{5}$. \\ Florian Wegwitz $\mathbb{D}^{2} \cdot$ Zhifu Sun $\mathbb{D}^{3} \cdot$ Michael Ghadimi $^{2} \cdot$ Rodney D. Newberry ${ }^{6} \cdot$ R. Balfour Sartor ${ }^{5}$. \\ William A. Faubion ${ }^{1} \cdot$ Steven A. Johnsen $\mathbb{1}^{2,7}$
}

Received: 3 December 2020 / Revised: 7 May 2021 / Accepted: 12 May 2021 / Published online: 4 June 2021

(c) The Author(s) 2021. This article is published with open access

\begin{abstract}
Despite the identification of several genetic factors linked to increased susceptibility to inflammatory bowel disease (IBD), underlying molecular mechanisms remain to be elucidated in detail. The ubiquitin ligases RNF20 and RNF40 mediate the monoubiquitination of histone $\mathrm{H} 2 \mathrm{~B}$ at lysine $120(\mathrm{H} 2 \mathrm{Bub} 1)$ and were shown to play context-dependent roles in the development of inflammation. Here, we aimed to examine the function of the RNF20/RNF40/H2Bub1 axis in intestinal inflammation in IBD patients and mouse models. For this purpose, intestinal sections from IBD patients were immunohistochemically stained for H2Bub1. Rnf20 or Rnf40 were conditionally deleted in the mouse intestine and mice were monitored for inflammation-associated symptoms. Using mRNA-seq and chromatin immunoprecipitation (ChIP)-seq, we analyzed underlying molecular pathways in primary intestinal epithelial cells (IECs) isolated from these animals and confirmed these findings in IBD resection specimens using ChIP-seq.The majority (80\%) of IBD patients displayed a loss of H2Bub1 levels in inflamed areas and the intestine-specific deletion of Rnf2O or Rnf4O resulted in spontaneous colorectal inflammation in mice. Consistently, deletion of Rnf2O or Rnf40 promoted IBD-associated gene expression programs, including deregulation of various IBD risk genes in these animals. Further analysis of murine IECs revealed that H3K4me3 occupancy and transcription of the Vitamin D Receptor ( $V d r$ ) gene and VDR target genes is RNF20/40-dependent. Finally, these effects were confirmed in a subgroup of Crohn's disease patients which displayed epigenetic and expression changes in RNF20/40-dependent gene signatures. Our findings reveal that loss of H2B monoubiquitination promotes intestinal inflammation via decreased VDR activity thereby identifying RNF20 and RNF40 as critical regulators of IBD.
\end{abstract}

These authors contributed equally: Robyn Laura Kosinsky, Maria Zerche

These authors jointly supervised this work: William A. Faubion, Steven A. Johnsen

Edited by M. Oren

Supplementary information The online version contains supplementary material available at https://doi.org/10.1038/s41418021-00808-w.

Robyn Laura Kosinsky

kosinsky.robynlaura@mayo.edu

Steven A. Johnsen

johnsen.steven@mayo.edu

Extended author information available on the last page of the article

\section{Introduction}

Inflammatory bowel disease (IBD), including Crohn's disease (CD) and ulcerative colitis (UC), affects approximately one individual in 250 Europeans and North Americans [1]. Indeed, rapid increases in the incidence of IBD within populations strongly suggest an environmental component to disease susceptibility. One major link between the exposome and pathophysiology is chromatin modifications stably altering transcriptional programs and cellular function [2]. One such epigenetic modifier, the obligate RNF20/ RNF40 heterodimer responsible for H2B monoubiquitination (H2Bub1), has recently been implicated in intestinal inflammation in a context-dependent fashion [3, 4]. A recent CRISPR screen identified RNF20 as a negative modulator of regulatory $\mathrm{T}$ cells [5] which play an important 
role in the pathogenesis of IBD [6, 7]. In addition, the heterozygous global knockout of Rnf20 in mice resulted in increased infiltration of myeloid-derived suppressor cells [4]. Thus, while the H2B monoubiquitination pathway is clearly essential for immune regulation, little mechanistic detail is known about the cell-intrinsic function of RNF20 and RNF40 in IBD.

Generally, the obligate RNF20/RNF40 heterodimer is recruited by the WW domain-containing adapter protein with coiled-coil (WAC) to the elongating RNA Polymerase II (RNAPII) following phosphorylation of serine 2 within the C-terminal domain of RNAPII [8]. As an E3 ligase complex, the RNF20/40 dimer monoubiquitinates histone $\mathrm{H} 2 \mathrm{~B}$ at lysine 120, an epigenetic mark associated with increased chromatin accessibility, resulting in eased passage of RNAPII and highly active transcriptional elongation [9]. In addition, as supported by studies in yeast [10,11] and our own experiments [12], H2Bub1 regulates the trimethylation of histone 3 at lysine 4 (H3K4me3), which is also tightly associated with transcriptional elongation [13]. Epigenetic pathways marked by $\mathrm{H} 3 \mathrm{~K} 4 \mathrm{me} 3$ occupancy are emerging as relevant therapeutic targets in IBD patients as evidenced by recent studies $[14,15]$.

Besides its role in transcriptional regulation, H2Bub1 was suggested as a potential tumor-suppressive marker in colorectal cancer (CRC) [16]. Counterintuitively, depletion of RNF20 and RNF40 resulted in reduced tumorigenic potential of CRC cells in vitro, potentially due to enhanced apoptosis rates [3, 17]. In addition, ablation of either, RNF20 or RNF40, modulates Nuclear Factor kappa-light-chain-enhancer of activated B cells (NF- $\mathrm{KB}$ ) signaling [3, 4, 18]. However, the consequences of Rnf2O and Rnf40 loss in the context of intestinal inflammation have not been directly compared in the same experimental settings.

Given the scarce knowledge on the cell-specific functions of RNF20/RNF40, we sought to investigate the importance of the $\mathrm{H} 2 \mathrm{~B}$ monoubiquitination pathway in IBD through a multidimensional analysis of mouse to human experimental systems. We detected reduced H2Bub1 amounts in $80 \%$ of IBD patients and, accordingly, intestinal deletion of Rnf 20 or Rnf40 resulted in the development of spontaneous colorectal inflammation in mice. Of mechanistic importance, we found Vitamin D Receptor ( Vdr) gene as well as VDR target gene H3K4me3 occupancy and expression to be highly dependent upon RNF20/40. As vitamin $\mathrm{D}$ status remains one of the few environmental associations with IBD activity [19], this report provides unique insight into the epigenetic regulation of this key IBD-associated factor.

\section{Results}

\section{Loss of H2Bub1 is associated with intestinal inflammation in IBD patients}

A previous mouse model of global haploinsufficiency of Rnf 20 suggested that the H2B monoubiquitination pathway may suppress intestinal inflammation [4]. However, there is a lack of knowledge on cell-specific functions of RNF20 and RNF40 in IBD. To examine a potential cell typespecific function of these $\mathrm{H} 2 \mathrm{~B}$ ubiquitin ligases, we analyzed a recent single cell RNA-seq dataset comparing cell-specific transcriptome-wide effects in Crohn's disease (CD) patients [20]. Upon clustering of cell types (Fig. 1A), we detected RNF2O and RNF4O gene expression primarily within epithelial cells (Fig. 1B). Therefore, to test for a putative function of the RNF20/40 axis in controlling intestinal inflammation, we studied colon sections of dextran sodium sulfate (DSS)-treated mice by performing immunohistochemistry for H2Bub1. Interestingly, H2Bub1 was lost in the majority of inflamed epithelial areas (Fig. 1C, D). To evaluate, whether this finding could be translated to the human condition, we performed immunohistochemical staining of H2Bub1 in a panel of human Crohn's disease samples with corresponding uninflamed adjacent tissue (NAT) from resection margins as a control. Notably, when quantifying nuclear staining intensity, we observed a significant decrease in H2Bub1 levels in inflamed epithelium (Fig. 1E, F). In fact, when comparing inflamed to healthy tissue within the same individual, we discovered that $80 \%$ of IBD patients displayed a strong reduction in H2Bub1-positive inflamed epithelial cells, while only $20 \%$ of patients displayed no changes in H2Bub1 levels. The observed association between H2Bub1 deficiency and intestinal inflammation led us to subsequently investigate the in vivo mechanistic role of RNF20/ 40 in the intestinal epithelium.

\section{Genetic deletion of $R n f 20$ or $R n f 40$ promotes spontaneous colorectal inflammation}

To evaluate the biological and molecular consequences of H2Bub1 loss in intestinal epithelial cells, we generated Villin-CreER ${ }^{\mathrm{T} 2}$, Rnf $20^{\text {flox, }}$ and Villin-CreER ${ }^{\mathrm{T} 2}, R n f 40^{\text {flox }}$ mice (Fig. 1G). The conditional tamoxifen-inducible deletion of each of the E3 ligases Rnf2O and Rnf40 individually provided complementary approaches to induce the loss of H2Bub1 in intestinal epithelial cells. Notably, 14 days after the first tamoxifen injection, $R n f 20^{\mathrm{f} / \mathrm{fl}}$ and $R n f 40^{\mathrm{f} / \mathrm{fl}}$ mice already displayed severe weight loss of up to ca. $15 \%$ (Fig. 1H, Supplementary Fig. S1A). Accordingly, the 
A
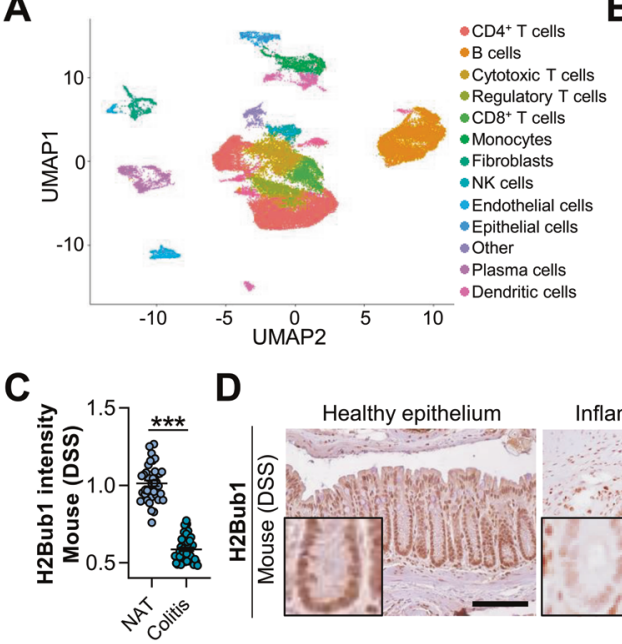

$\mathbf{F}$

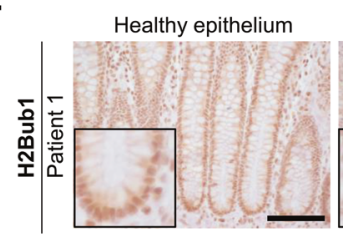

G

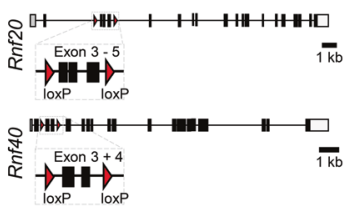

Inflamed epithelium

H
B

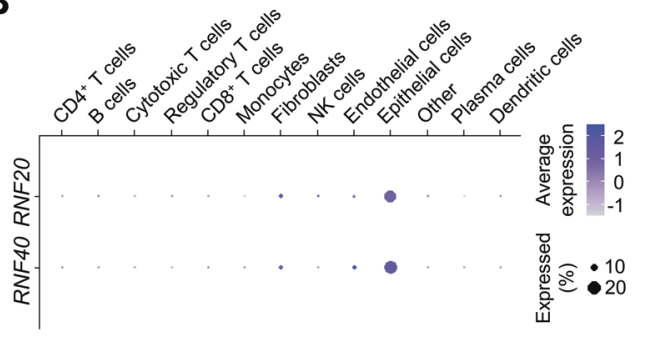

E
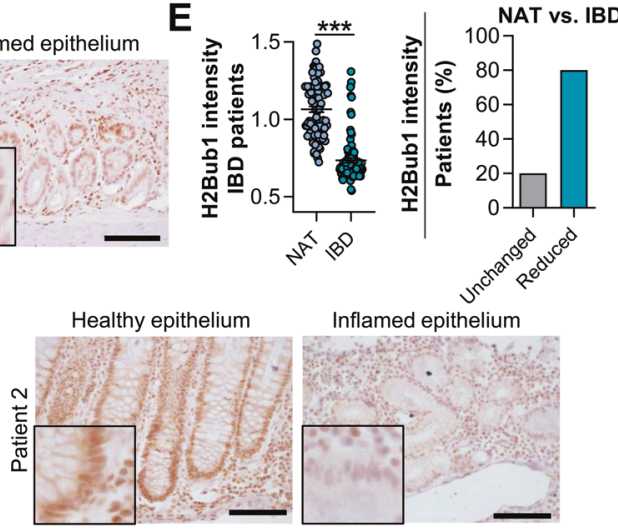

Inflamed epithelium

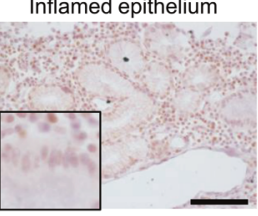

I

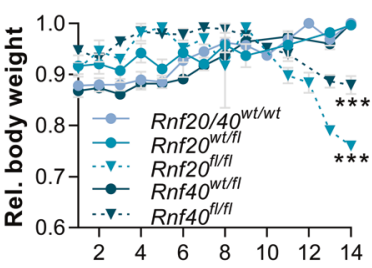

Days after Tamoxifen

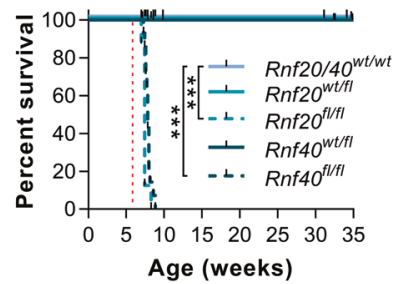

Fig. 1 IBD patients display H2Bub1 loss in inflamed intestinal epithelium. A Cell type clustering in a uniform manifold approximation and projection (UMAP) based on scRNA-seq data evaluating cell-specific gene expression changes between uninflamed and inflamed resection specimens isolated from Crohn's disease patients [20]. B Dot plot displaying the expression level and the percentage of cells within each cell cluster expressing RNF2O and RNF40. RNF2O and $R N F 40$ levels were highest in epithelial cells. C Colon sections of C57BL/6N mice treated with $0.75 \%$ DSS $(n=6)$ for 14 days were stained for H2Bub1 using IHC. Relative staining intensity was quantified using FIJI in five images per mouse. D Representative images of murine colon sections displaying reduced H2Bub1 levels in inflamed epithelium. Scale bar: $100 \mu \mathrm{m}$. E Intestinal sections of Crohn's disease patients $(n=18)$ were stained for H2Bub1 using IHC. Relative

Disease Activity Index (DAI) was elevated in these animals (Supplementary Fig. S1B-E). As a consequence of high disease activity, $R n f 20^{\mathrm{f} / \mathrm{fl}}$ and $R n f 40^{\mathrm{A} / \mathrm{fl}}$ mice displayed significantly decreased survival compared to wild type and heterozygous litter mates (Fig. 1I).

Consistent with an increased DAI and reduced survival, mice with an intestinal deletion of Rnf 20 or Rnf40 displayed significantly decreased colon length (Fig. 2A, Supplementary Fig. S1F, G). After confirming the successful loss of H2Bub1, RNF20, and RNF40 in $R n f 20^{\mathrm{f} / \mathrm{fl}}$ and $R n f 40^{\mathrm{f} / \mathrm{fl}}$ animals (Fig. 2B, Supplementary Fig. S1H), H\&E staining staining intensity was quantified using FIJI in five images per patient. When comparing healthy resection margins to inflamed tissue, $80 \%$ of patients displayed reduced H2Bub1 levels in inflamed regions. F Representative images of colon sections of two IBD patients stained for H2Bub1. Scale bar: $100 \mu \mathrm{m}$. G Mice with loxP-flanked exons 3-5 of Rnf20 or loxP-flanked exons 3-4 of Rnf40 were mated with Villin$\mathrm{CreER}^{\mathrm{T} 2}$ mice to enable a tamoxifen-inducible intestinal knockout of $R n f 20$ or Rnf40, respectively. H Fourteen days after the first tamoxifen injection, $R n f 20^{\mathrm{f} / \mathrm{fl}}$ and $R n f 40^{\mathrm{f} / \mathrm{fl}}$ mice displayed severe weight loss and, therefore, (I) significantly reduced survival (red dotted line represents start of tamoxifen treatment). Survival data were based on local and institutional guidelines according to which animals needed to be sacrificed after a weight loss of $20 \%$. One-way ANOVA, mean \pm SEM.

of the colon revealed severe epithelial damage in Rnf20- and Rnf40-deficient mice (Fig. 2C). The colorectal epithelial damage was quantified by determination of the $\mathrm{H}$-score, a scoring system based on the intactness of the colonic epithelium and lymphocyte infiltration. Indeed, $R n f 20^{\mathrm{A} / \mathrm{fl}}$ and $R n f 40^{\mathrm{f} / \mathrm{fl}}$ mice displayed a high degree of epithelial damage and lymphoid infiltrates, contributing to a significantly increased H-score (Fig. 2D, E; Supplementary Fig. S1I). Consistently, we observed increased infiltration of CD45positive immune cells (Fig. 2F, G) and increased vascularization (Supplementary Fig. S1J, K) in these animals while 
Fig. 2 Intestinal $R n f 20$ and

Rnf40 deletion results in spontaneous colorectal inflammation in mice. A Homozygous Rnf 20 or Rnf40 deletion resulted in decreased colon length. B The knockout efficiency in $R n f 20^{\mathrm{f} / \mathrm{fl}}$ and $R n f 40^{\mathrm{fl} / \mathrm{fl}}$ mice was demonstrated using IHC for $\mathrm{H} 2 \mathrm{Bub} 1$ on colon sections. C Colons were H\&Estained and (D) the percentage of segments with mild [1], medium [2], or severe [3] epithelial damage was determined. Rnf 20 and Rnf40 intestine-specific knockout mice displayed more areas with epithelial damage than wildtype or heterozygous animals.

E Based on the damage, the

$H$ score was calculated and shown to be elevated upon the loss of Rnf2O and Rnf40. F IHC for CD45-positive cells in the colon revealed $(\mathbf{G})$ increased infiltration of immunoregulatory cells. Scale bar: $100 \mu \mathrm{m}$. Oneway ANOVA, mean \pm SEM.
A
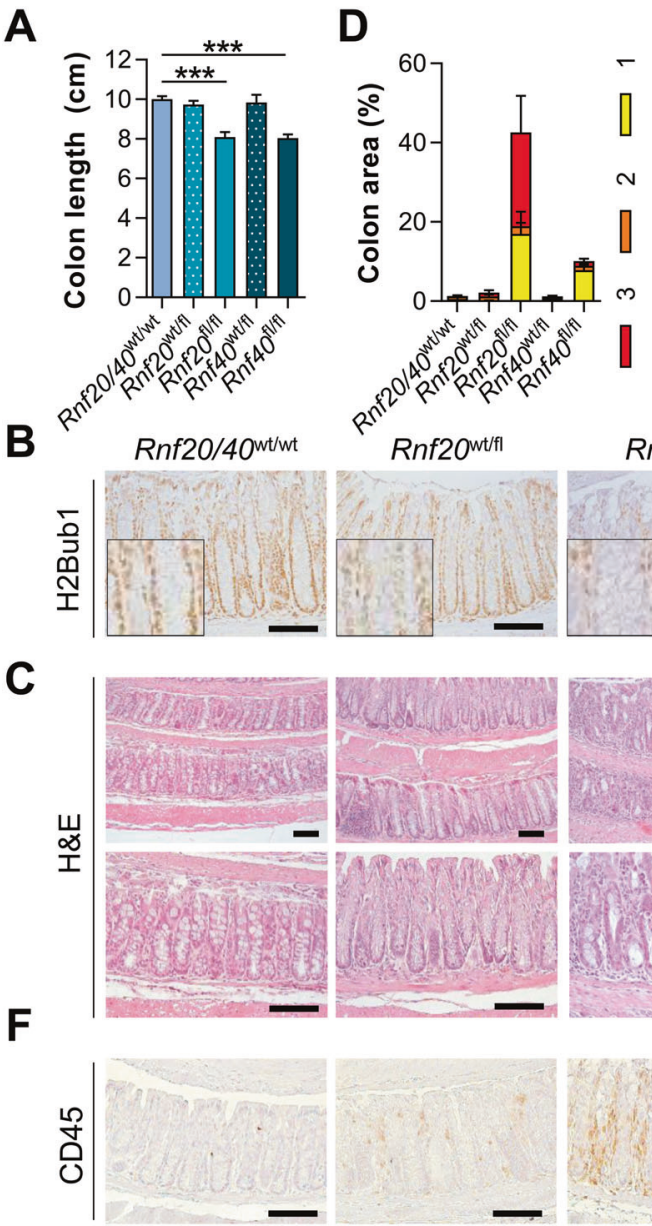
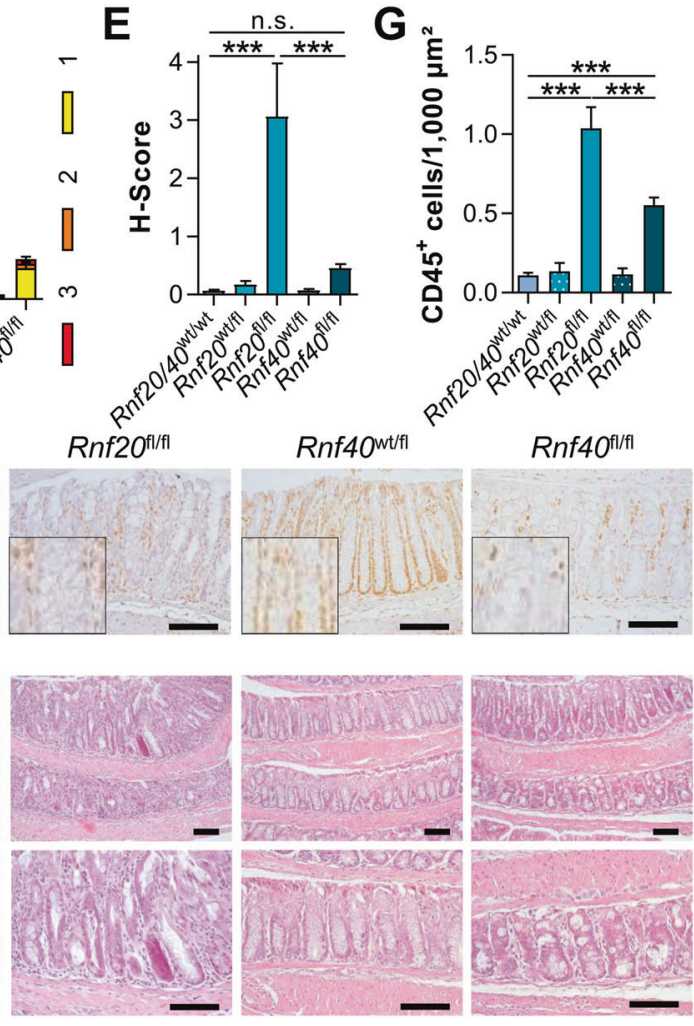

G
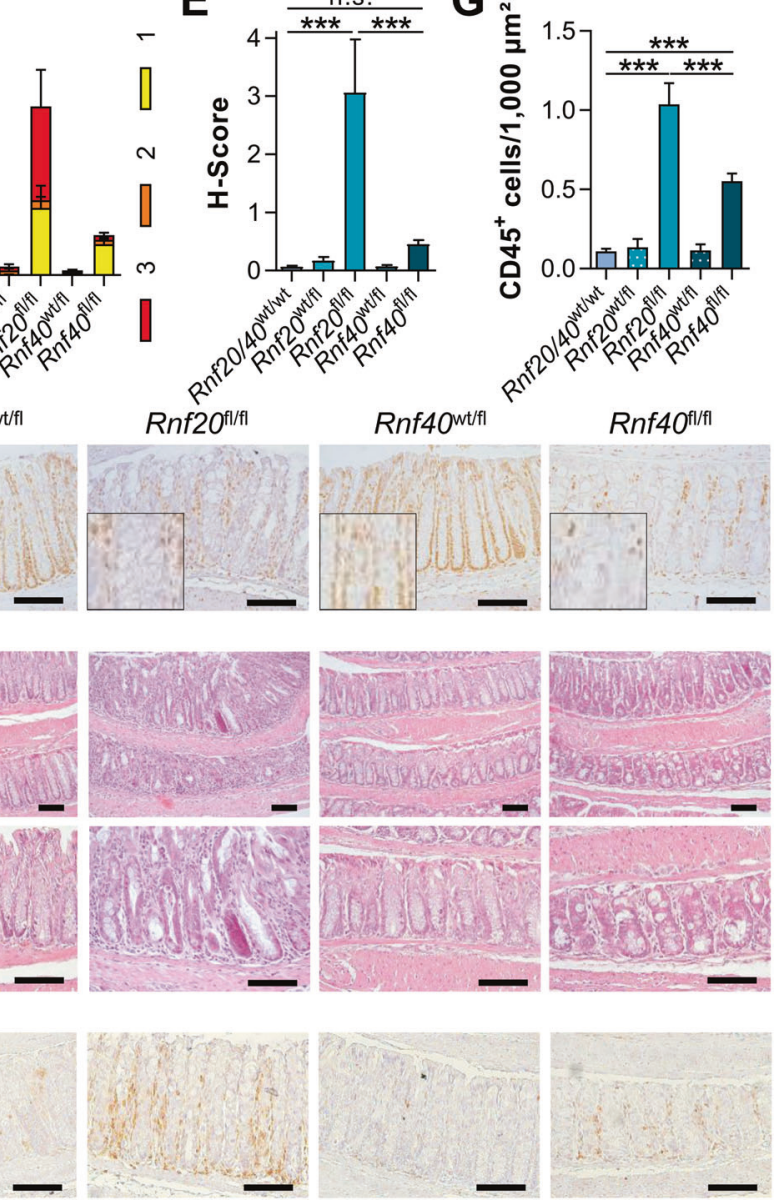

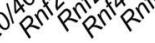

only minor effects were observed on serum IL-6 levels (Supplementary Fig. S1L). In summary, the Villin-CreER ${ }^{\mathrm{T} 2}$ mediated deletion of $R n f 20$ or $R n f 40$ promotes spontaneous colorectal inflammation in mice. In contrast, mice heterozygous for Rnf20 or Rnf40 deletion did not develop spontaneous inflammation (Supplementary Fig. S2) and did not display increased sensitivity to DSS-mediated colitis (Supplementary Fig. S3). These results suggest that only a complete loss of $R n f 2 O$ and $R n f 40$, and therefore H2Bub1, is sufficient to induce spontaneous colorectal inflammation.

\section{Rnf20/40 deletion promotes IBD-associated gene expression programs}

To gain initial insight into the molecular mechanisms by which perturbation of the $\mathrm{H} 2 \mathrm{~B}$ monoubiquitination axis leads to intestinal inflammation, we examined transcriptomewide changes in intestinal epithelial cells (IECs) directly isolated from the colons of Villin-CreER ${ }^{\mathrm{T} 2}, R n f 20^{\mathrm{fl} / \mathrm{fl}}$ and $R n f 40^{\mathrm{f} / \mathrm{fl}}$ mice and compared these to wild type controls 14 days after tamoxifen injection. After confirming the loss of $R n f 20$ and Rnf40 expression at the mRNA and protein level as well as the loss of H2Bub1 (Fig. 3A, B), we performed mRNA-seq. As expected, Rnf20 and Rnf40 deletion resulted in the differential regulation of a broad variety of genes (Fig. 3C). Gene subsets were divided into three clusters (upregulated in wild type, $R n f 20^{\mathrm{fl} / \mathrm{fl}}$ or $R n f 40^{\mathrm{f} / \mathrm{fl}}$, respectively) and analyzed for pathway enrichment using Enrichr [21, 22]. Interestingly, factors involved in $\mathrm{O}$ glycosylation of mucins and other proteins were commonly downregulated upon loss of $R n f 20$ or Rnf40. While many processes were commonly enriched following deletion of either H2B ubiquitin ligase, $R n f 20^{\mathrm{f} / \mathrm{fl}}$ IECs demonstrated a higher enrichment of gene sets related to cytokine signaling and epidermal differentiation while $R n f 40^{\mathrm{fl} / \mathrm{fl}}$ cells showed an enrichment of ribosome biogenesis and gene expressionrelated processes (Fig. 3D). Finally, we sought to determine whether gene expression patterns altered following Rnf20 and Rnf40 deletion in IECs may be relevant for murine models for colitis and human IBD. Indeed, we found that genes related to inflamed tissue in DSS-treated mice [23] as well as in patients with severe IBD [24] were enriched in $R n f 20^{\mathrm{f} / \mathrm{fl}}$ and $R n f 40^{\mathrm{f} / \mathrm{fl}}$ IECs (Fig. 3E). Thus, the intestinal deletion of Rnf20 and Rnf40 in murine IECs is associated with gene expression profiles induced in murine and human intestinal inflammation. 


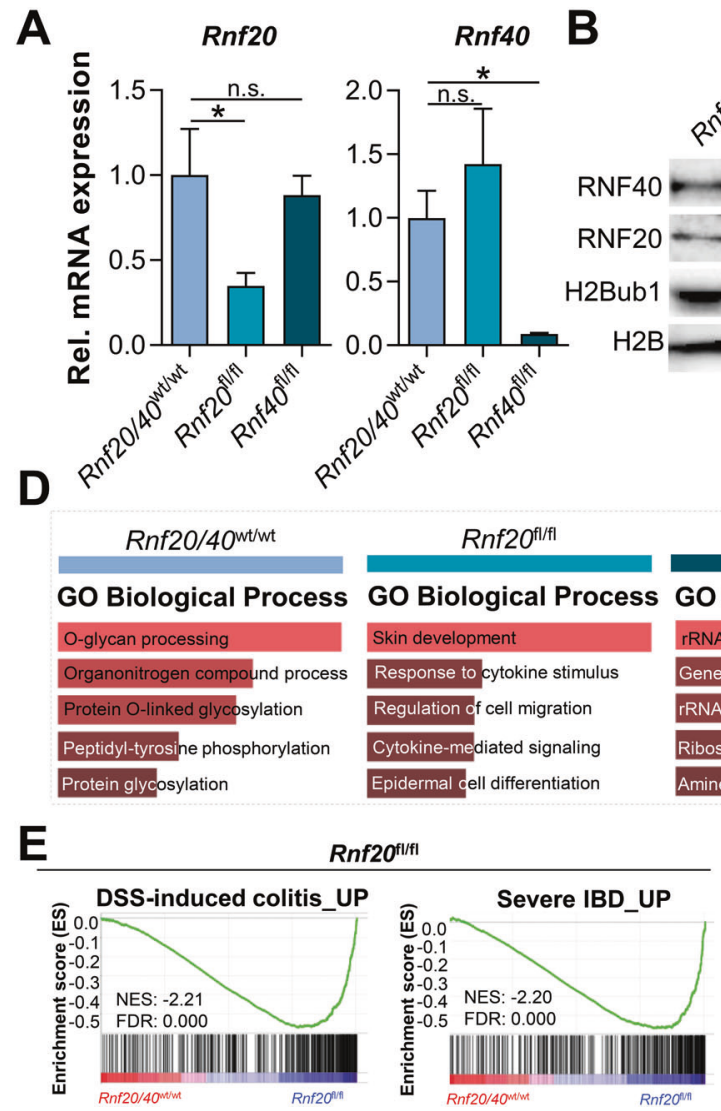

Fig. 3 The intestinal loss of either Rnf20 or Rnf40 drives IBDassociated transcriptome-wide changes. A The loss of $R n f 20$ or Rnf40 in IECs was confirmed at the mRNA and (B) protein levels using qRT-PCR and western blot, respectively. C mRNA-seq was performed using wild type, $R n f 20^{\mathrm{f} / \mathrm{fl}}$ and $\operatorname{Rnf} 40^{\mathrm{f} / \mathrm{fl}}$ IECs $(n=2)$. Heatmap depicting the transcriptome-wide effects of Rnf20 and Rnf40 deletion by showing significantly (padj $\leq 0.05)$ upregulated $(\log 2 \mathrm{FC} \geq$ 1 , red) or downregulated ( $\log 2 \mathrm{FC} \leq-1$, blue) genes. D Based on expression patterns, genes were divided into three clusters which were

\section{RNF20 and RNF40 are required for the expression of IBD susceptibility genes}

Early studies exploring the molecular function of H2Bub1 revealed that this epigenetic mark is highly enriched across the transcribed region of active genes [25]. Mechanistically, H2Bub1 enables RNAPII passage through chromatin [26]. $\mathrm{H} 2 \mathrm{Bub} 1$ also facilitates deposition of $\mathrm{H} 3 \mathrm{~K} 4 \mathrm{me} 3$ in yeast $[10,11]$. The histone modification $\mathrm{H} 3 \mathrm{~K} 4 \mathrm{me} 3$ is an established mark of actively transcribed transcription start sites (TSS) and gene bodies. Indeed, our prior work substantiated a role for $\mathrm{H} 2 \mathrm{Bub} 1$ in elongation-associated spreading of H3K4me3 into the gene body of RNF40-dependent genes [12]. Thus, we performed ChIP-seq in IECs to analyze H3K4me3 occupancy upon the deletion of Rnf 20 and $R n f 40$. Consistent with our prior work, loss of $R n f 20$ or Rnf4O resulted in a significant reduction of $\mathrm{H} 3 \mathrm{~K} 4 \mathrm{me} 3$ occupancy in the gene body with only marginal effects closer to the TSS (Fig. 4A). Focusing on this discovery, we closely examined the genes displaying RNF20/40-dependent changes in H3K4me3 occupancy and decreased expression. Given the inflammatory consequences of an intestine-specific $R n f 20 / 40$ deletion in mice, we performed an enrichment analysis of the RNF20/40-dependent genes with published IBD susceptibility gene lists [27, 28]. Remarkably, $19 \%$ of published IBD susceptibility genes appeared to be regulated by this RNF20/RNF40/H3K4me3dependent mechanism (Fig. 4B). Consistently, a downregulation of those IBD susceptibility genes at the mRNA level could be confirmed in our mRNA-seq data (Fig. 4C). In summary, these findings reveal a previously unknown function of RNF20/40 in regulating H3K4me3 occupancy and gene expression of a high proportion of IBD susceptibility genes. 


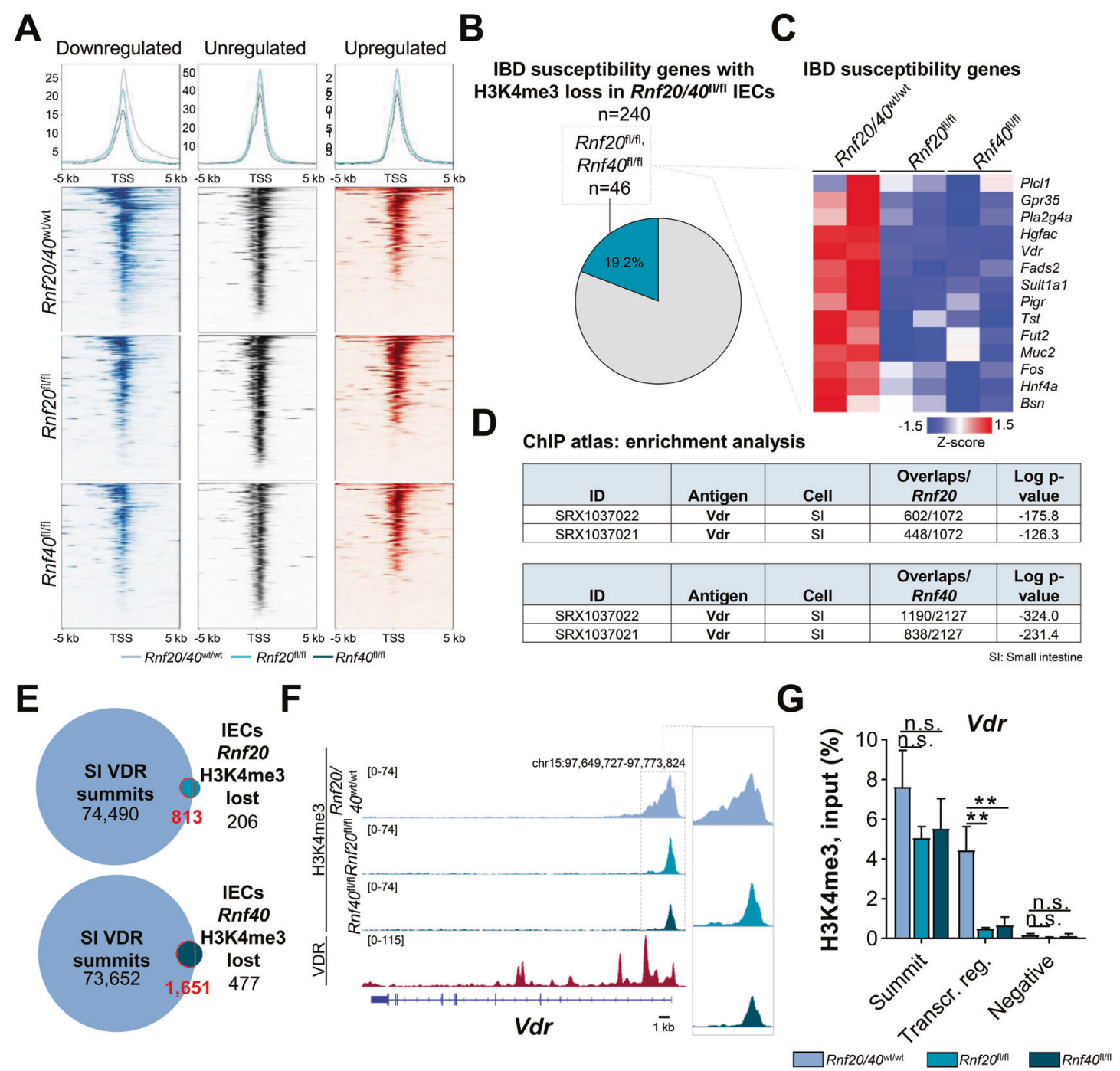

Fig. 4 Loss of RNF20 or RNF40 causes reduced H3K4me3 occupancy on IBD risk genes including $\boldsymbol{V} \boldsymbol{d r}$. A Average binding profiles and heatmaps depicting occupancy (RPKM values) of H3K4me3 around the TSS $( \pm 5 \mathrm{~kb})$ of genes upregulated, unregulated and downregulated in wild type, $R n f 20^{\mathrm{f} / \mathrm{fl}}$ and $R n f 40^{\mathrm{f} / \mathrm{fl}}$ IECs. Genes were sorted high to low based on H3K4me3 occupancy under wild type conditions. Binding profiles revealed decreased H3K4me3 occupancy specifically in the gene body after Rnf 20 or Rnf 40 loss leading to peak narrowing. B Genes which lost H3K4me3 occupancy in $R n f 20^{\mathrm{f} / \mathrm{fl}}$ and $R n f 40^{\mathrm{f} / \mathrm{fl}}$ IECs were compared for overlap with IBD susceptibility genes [27, 28] expressed in IECs. H3K4me3 occupancy of $19 \%$ of IBD risk genes is dependent on RNF20 and/or RNF40. C Heatmap depicting gene expression patterns of a subset of IBD susceptibility genes in wild type, Rnf $20^{\mathrm{f} / \mathrm{fl}}$ and $R n f 40^{\mathrm{f} / \mathrm{fl}}$ IECs (padj $\leq 0.05$, red: upregulated $(\log 2 \mathrm{FC} \geq 1)$, blue: downregulated $(\log 2 \mathrm{FC} \leq-1)$ ).

\section{H3K4me3 occupancy on Vdr and VDR target genes is RNF20- and RNF40-dependent}

In order to uncover further upstream regulatory mechanisms controlling the expression of RNF20/40-dependent genes in IEC, we examined whether specific transcription factors
D Regions that lost H3K4me3 occupancy in Rnf2O and Rnf40 null IECs were compared to previously published ChIP-seq experiments using ChIP Atlas (http://chip-atlas.org) displaying a high overlap between our datasets and VDR-bound regions in the murine small intestine (SI) [29]. E By analyzing publicly available VDR ChIP-seq data, VDR-bound regions in murine SI were identified $(75,303$ regions) [29] and compared to regions displaying decreased H3K4me3 occupancy in $\operatorname{Rnf} 20$ (1,019 regions) and $R n f 40$ (2,128 regions) knockout IECs. $79.8 \%$ (813/1,019 regions) of Rnf2O- and $77.6 \%$ (1,651/2,128 regions) of Rnf40-dependent H3K4me3 regions were also occupied by VDR. F Binding profiles of VDR in SI [29] and $\mathrm{H} 3 \mathrm{~K} 4 \mathrm{me} 3$ in IECs revealed $\mathrm{H} 3 \mathrm{~K} 4 \mathrm{me} 3$ peak narrowing under Rnf 20 or Rnf40 knockout conditions. G Reduced H3K4me3 occupancy in the $V d r$ gene body in $R n f 20^{\mathrm{f} / \mathrm{fl}}$ and $R n f 40^{\mathrm{f} / \mathrm{fl}}$ IECs was confirmed by ChIPqPCR $(n=4)$. One-way ANOVA, mean \pm SEM.

displayed a particular enrichment on these genes using occupancy data from publically available ChIP-seq datasets using ChIP Atlas (http://chip-atlas.org). Intriguingly, we found Vitamin D Receptor (VDR)-bound regions [29] (small intestine, mouse, Fig. 4D, E) to be significantly enriched within our dataset. Validating our bioinformatic 
A

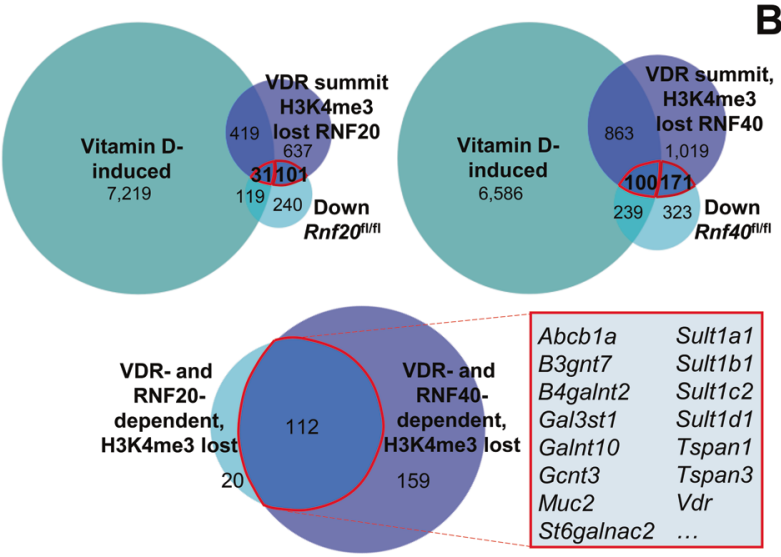

B

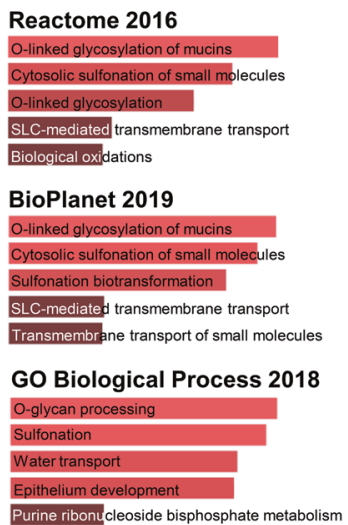

C

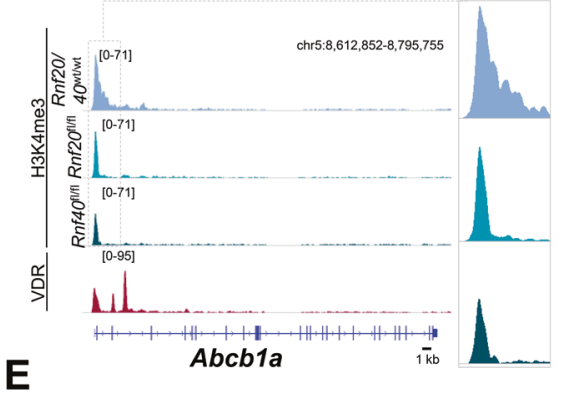

D
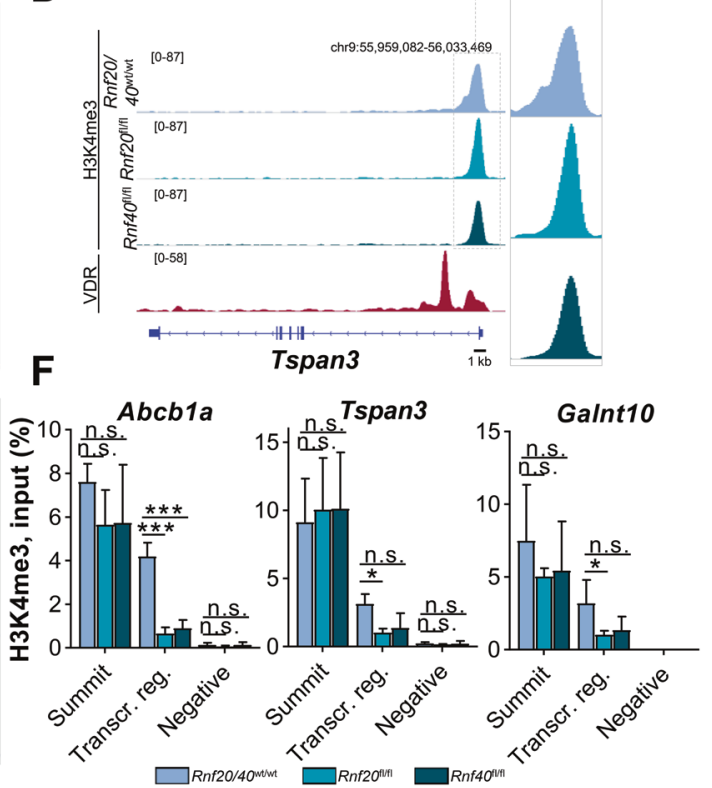

Fig. 5 RNF20 and RNF40 are required for transcriptional elongation-associated H3K4me3 spreading on VDR target genes. A Venn diagram displaying the overlap between vitamin D-induced genes [29], genes downregulated following Rnf20 or Rnf40 knockout in IECs identified by mRNA-seq and VDR-dependent genes displaying reduced H3K4me3 occupancy upon Rnf2O or Rnf4O deletion in IECs (upper panel). Numbers marked in red were overlapped to identify VDR- and RNF20/40-dependent genes with reduced H3K4me3 occupancy (lower panel). B Enrichr gene ontology analysis

methodology, the $V d r$ gene itself was bound by VDR and displayed profound $\mathrm{H} 3 \mathrm{~K} 4 \mathrm{me} 3$ peak narrowing following the loss of $R n f 20$ or Rnf40 (Fig. 4F). This finding was further confirmed using ChIP-qPCR, where a significant reduction of $\mathrm{H} 3 \mathrm{~K} 4 \mathrm{me} 3$ occupancy was observed in the transcribed region of the $V d r$ gene (Fig. 4G). To obtain further insight into the relationship between RNF20/40- and VDR-dependent gene regulation, genes induced in the small intestine of vitamin D-treated mice [29] were overlapped with genes downregulated upon $R n f 20$ or Rnf40 loss (mRNA-seq) and genes which displayed decreased of these 112 genes revealed their involvement in O-linked glycosylation and sulfonation of proteins. $\mathbf{C}-\mathbf{E}$ ChIP-seq tracks of three exemplary genes (Abcb1a, Tspan3, Galnt10) illustrated H3K4me3 peak narrowing in Rnf2O and Rnf40 knockout IECs and VDR occupancy in murine small intestine [29]. F ChIP-qPCR for Abcbla, Tspan 3 and Galnt 10 was performed using primers complementary to the peak summit, in the transcribed region and a negative control site. Upon Rnf20 and Rnf40 deletion, occupancy in the transcribed region was significantly decreased $(n=4)$. One-way ANOVA, mean \pm SEM.

H3K4me3 occupancy in Rnf2 $0^{\mathrm{f} / \mathrm{fl}}$ and $R n f 40^{\mathrm{f} / \mathrm{fl}}$ IECs (ChIPseq). Genes commonly regulated by vitamin D, RNF20, and RNF40 included several IBD susceptibility genes as well as mucin-modifying factors (Fig. 5A, B). Indeed, ChIP-seq tracks (Fig. 5C-E) and ChIP-qPCR (Fig. 5F) confirmed that these genes displayed reduced $\mathrm{H} 3 \mathrm{~K} 4 \mathrm{me} 3$ occupancy specifically in their transcribed regions. Together, using corroborating methodology, these results indicate that RNF20 and RNF40 loss reduces H3K4me3 occupancy on the IBD susceptibility gene $V d r$ as well as VDR target genes. 


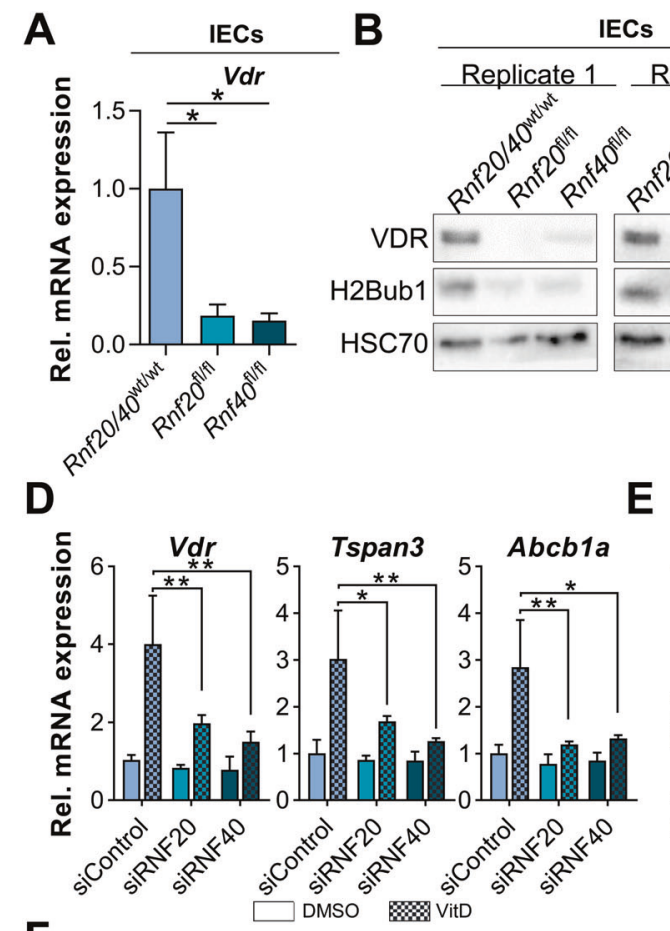

$\mathbf{F}$
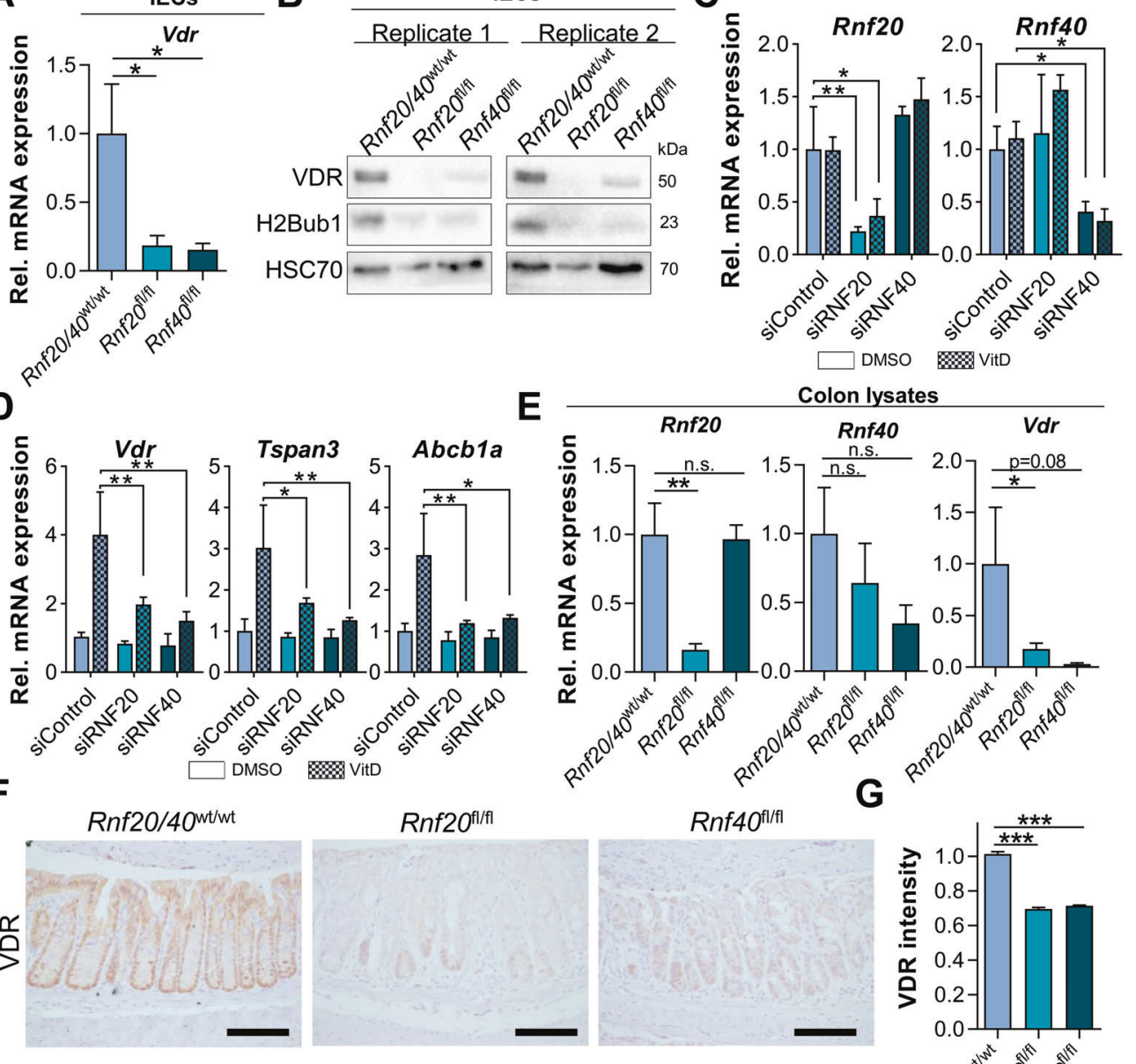

G

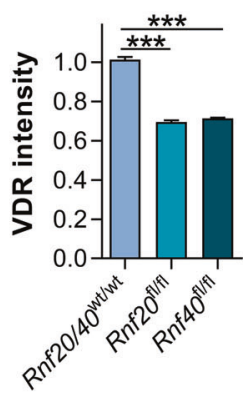

Fig. 6 RNF20 and RNF40 deficiency results in reduced VDR levels. A $R n f 20$ and $R n f 40$ deletion reduced $V d r$ mRNA and (B) protein levels in IECs as demonstrated by qRT-PCR and Western blot. C IECs were isolated from wild type mice and cultured under vitamin D-deficient conditions. RNF20 and RNF40 were transiently depleted using siRNA and $24 \mathrm{~h}$ after knockdown cells were treated with calcitriol $(1 \mu \mathrm{M})$ for $48 \mathrm{~h}(n=3)$. D Calcitriol treatment induced the

\section{The IBD risk gene $V d r$ is downregulated upon $R n f 20$ or Rnf40 deletion}

We observed that the IBD susceptibility gene $V d r$ encoding the vitamin D receptor was downregulated in our mRNAseq data and, indeed, we confirmed this finding in IECs at the mRNA and protein levels (Fig. 6A, B). Accordingly, the expression of VDR target genes was also significantly reduced in $R n f 20^{\mathrm{f} / / \mathrm{ll}}$ and $R n f 40^{\mathrm{f} / \mathrm{fl}}$ IECs (Supplementary Fig. S4A). Notably, the overexpression of human VDR in IECs rescued the downregulation of $V d r$ as well as VDRdependent genes upon the depletion of RNF20 and RNF40 (Supplementary Fig. S4B-C). Thus, multiple independent methodologies support that RNF20/40 regulates $V d r$ and
mRNA expression of VDR target genes in control cells but not in RNF20- and RNF40-depleted IECs as detected using qRT-PCR. E The reduction of $V d r$ following $R n f 20$ and $R n f 40$ genetic deletion was confirmed in whole colon lysates using qRT-PCR $(n=6)$. F IHC for VDR and subsequent (G) determination of staining intensity using FIJI verified reduced VDR protein amounts in $R n f 20^{\mathrm{f} / \mathrm{fl}}$ and $R n f 40^{\mathrm{f} / \mathrm{fl}}$ colons. Scale bar: $100 \mu \mathrm{m}$. One-way ANOVA, mean \pm SEM.
VDR gene targets by modulating H3K4 trimethylation and target gene transcription.

Next, we directly tested this mechanism through a reductionist in vitro approach. As our results were based on a vitamin D-proficient mouse model, we aimed to verify our hypothesis that RNF20 and RNF40 are required to promote expression of $V d r$ and VDR target genes in another system. Thus, we isolated IECs from wild type animals and grew them in 2D cell culture under vitamin D-deficient conditions. Cells were transfected with siRNAs to transiently deplete RNF20 and RNF40 and were treated with calcitriol, the active form of vitamin D. Notably, the induction of $V d r$ and VDR target gene expression upon vitamin D treatment was significantly impaired following RNF20 or RNF40 

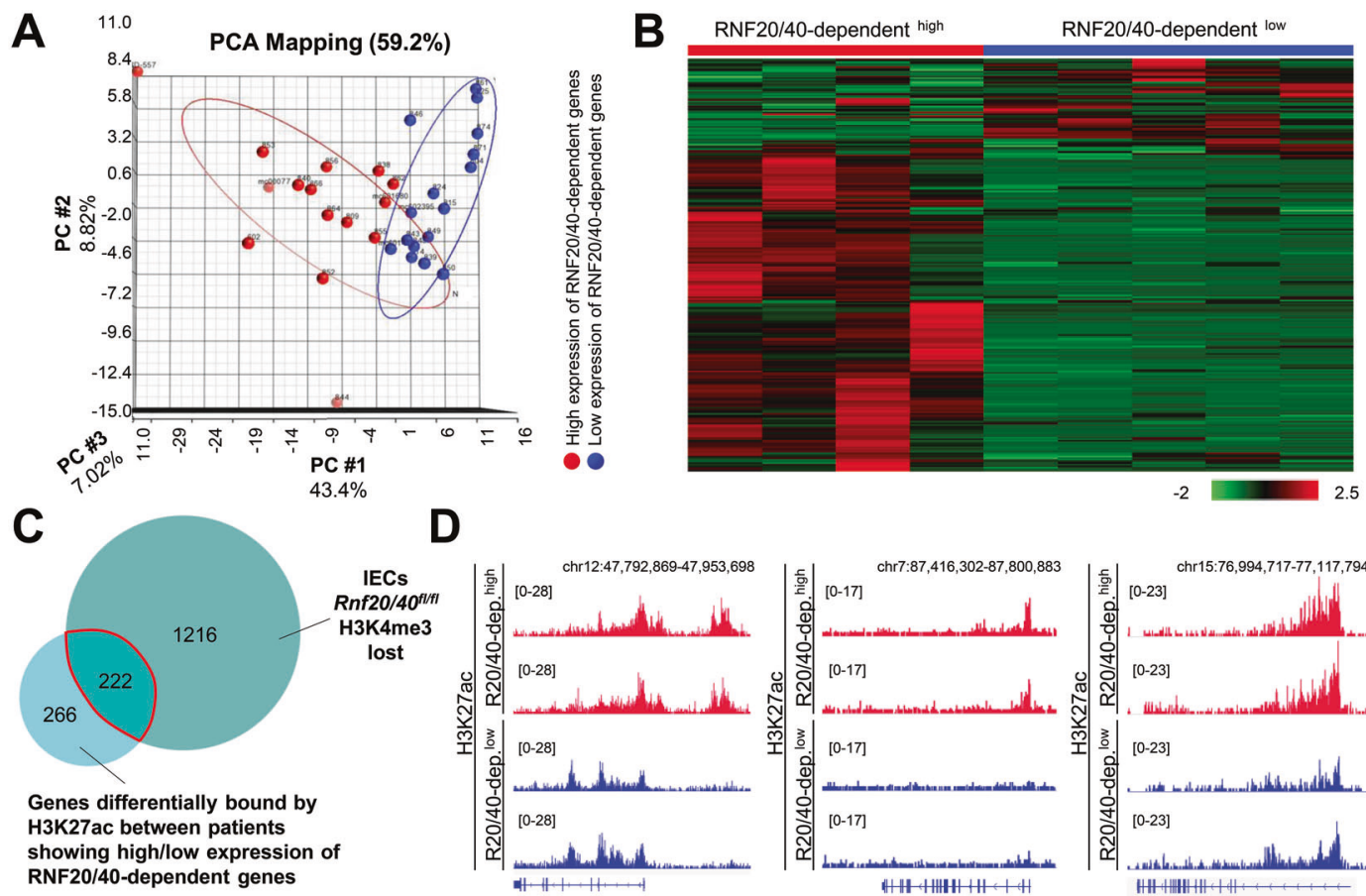

D
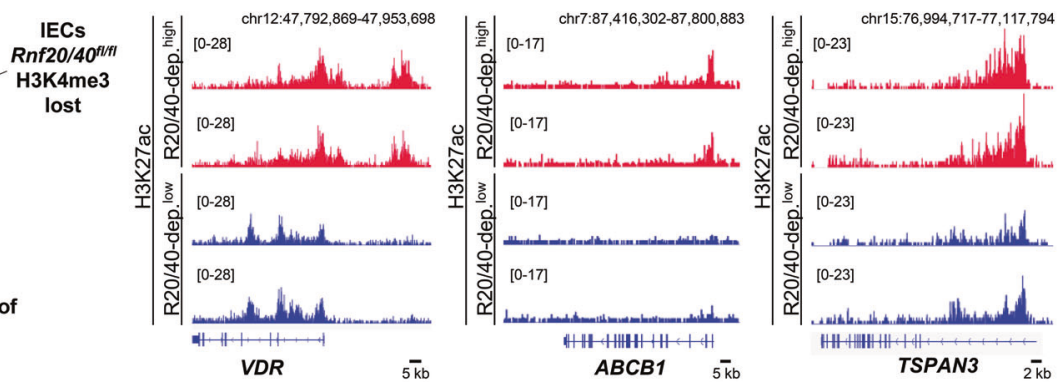

Fig. 7 RNF20/40-dependent genes are deregulated in a subset of IBD patients. A Principal component analysis demonstrating the separation of IBD patients $(n=30)$ displaying low (in blue) or high (in red) expression of RNF20/40-regulated genes detected by mRNA-seq in murine IECs. B Heatmap displaying the expression of genes differentially marked with $\mathrm{H} 3 \mathrm{~K} 27 \mathrm{ac}$ between a subset of IBD patients with markedly low $(n=5)$ or high $(n=4)$ expression of RNF20/40dependent genes. $\mathbf{C}$ Venn diagram showing the overlap between genes differentially bound by H3K27ac in IBD patients showing low or high expression of RNF20/40-regulated genes and genes which have lost H3K4me3 occupancy in Rnf20/40 $0^{\mathrm{f} / \mathrm{fl}}$ IECs. D Example occupancy profiles of $\mathrm{H} 3 \mathrm{~K} 27 \mathrm{ac}$ on $V D R, A B C B 1$, and TSPAN3 revealed decreased $\mathrm{H} 3 \mathrm{~K} 27 \mathrm{ac}$ occupancy in enhancer and promoter regions in IBD patients with low (blue) compared to high (red) expression of RNF20/40-dependent genes. depletion (Fig. 6C, D). We further confirmed the reduction of $V d r$ and VDR target genes in whole colon lysates isolated from $R n f 2 O^{\mathrm{fl} / \mathrm{fl}}$ and $R n f 40^{\mathrm{f} / / \mathrm{fl}}$ mice at the mRNA level (Fig. 6E, Supplementary Fig. S5A). Finally, we were able to verify that VDR protein levels and those of one of its targets were decreased upon Rnf2O and Rnf40 knockout via IHC on colon sections (Fig. 6F, G, Supplementary Fig. S5B, C).

\section{A subgroup of CD patients displays deregulation of RNF20/40-dependent gene signatures}

We have demonstrated that intestinal Rnf20 and Rnf40 deletion results in spontaneous colorectal inflammation in mice as well as reduced levels of $V d r$ and its target genes. Given the established association of Vitamin D biology as a risk factor to Crohn's disease activity, a cohort of CD patients was analyzed for gene expression and H3K27ac occupancy. We utilized an emerging methodology of RNA/ chromatin extraction from FFPE tissue from terminal ileal resection specimens isolated from a well characterized cohort of CD patients. Confirming the importance of this pathway, a subgroup of individuals displayed consistently low expression for the majority of genes downregulated in $R n f 20^{\mathrm{f} / \mathrm{fl}}$ and $R n f 40^{\mathrm{f} / \mathrm{fl}}$ IECs (Fig. 7A, B). Next, genes differentially marked with H3K27ac between patients with low and high expression of RNF20/40-dependent genes were overlapped with genes which lost H3K4me3 occupancy in $R n f 20^{\mathrm{f} / \mathrm{fl}}$ and $R n f 40^{\mathrm{f} / \mathrm{fl}}$ IECs. Intriguingly, $45 \%$ of genes differentially marked by H3K27ac in IBD patients also displayed decreased H3K4me3 in Rnf20/40-deficient IECs (Fig. 7C). Indeed, patients with low expression of RNF20/ 40-dependent genes displayed reduced H3K27ac occupancy on the IBD risk gene $V D R$ as well as VDR targets such as $A B C B 1$ and TSPAN3 (Fig. 7D). Notably, these patient subsets did not display differences in serum vitamin D levels, similar to the situation observed in our mouse model (Supplementary Fig. S5D, E). Together, our findings demonstrate that RNF20 and RNF40 not only regulate the IBD susceptibility gene VDR but also a high proportion of VDR target genes in mice. Importantly, we identified a subset of IBD patients that displays deregulated expression and epigenetic marking of RNF20/40-dependent VDR targets. 


\section{Discussion}

Our findings uncover a novel role for RNF20 and RNF40 in maintaining $V d r$ and VDR target gene expression and suppression of intestinal inflammation. While decreased H2Bub1 levels have been linked to tumor progression and poor survival in several cancer entities, including CRC [16], we report for the first time that $\mathrm{H} 2 \mathrm{~B}$ monoubiquitination is reduced in inflamed areas in $80 \%$ of Crohn's disease patients. In support of this finding, we observed that complete intestine-specific ablation of Rnf 20 or Rnf40, the genes encoding the E3 ligases mediating $\mathrm{H} 2 \mathrm{~B}$ monoubiquitination, promotes spontaneous intestinal inflammation in mice. Molecularly, our findings demonstrate that RNF20/40 loss leads to a global narrowing of $\mathrm{H} 3 \mathrm{~K} 4 \mathrm{me} 3$ peaks, especially on VDR-dependent genes.

Vitamin D is thought to be an important factor in the development and perpetuation of IBD, particularly CD [30]. For example, vitamin D deficiency in IBD patients has been described in several studies and was associated with increased disease activity [19]. These findings were confirmed in an $I l 10$ knockout-based experimental model for murine colitis and, indeed, vitamin D supplementation ameliorated disease activity and reduced mortality [31]. The biological activity of vitamin $\mathrm{D}$ is mediated by the binding of its active metabolite 1,25-dihydroxyvitamin D3 (1,25 $(\mathrm{OH}) 2 \mathrm{D} 3$; calcitriol) to the vitamin D receptor [32]. Intriguingly, VDR gene polymorphisms have also been described to increase IBD susceptibility $[33,34]$ and VDR levels were found to be profoundly reduced in inflamed colonic biopsies isolated from IBD patients compared to healthy controls. Accordingly, the deletion of $V d r$ in gut epithelial cells resulted in severe intestinal inflammation in chemically induced colitis in mice while elevated $V d r$ expression protected animals from symptoms [35]. Despite these findings supporting an important role of vitamin D receptor activity in IBD, environmental or epigenetic modifiers regulating this pathway remained to be elucidated.

As originally shown in yeast $[10,11]$ and supported by our previous studies [12], H2B monoubiquitination regulates $\mathrm{H} 3 \mathrm{~K} 4 \mathrm{me} 3$, an epigenetic mark associated with increased transcription elongation rates [13]. Consistently, the loss of H2Bub1 following intestinal Rnf 20 or Rnf 40 deletion resulted in decreased $\mathrm{H} 3 \mathrm{~K} 4 \mathrm{me} 3$ occupancy in the transcribed region of various IBD-associated genes. Notably, upon H2Bub1 loss, we observed H3K4me3 narrowing on the $V d r$ gene as well as decreased $V d r$ mRNA and protein amounts. Consequently, the induction of VDRdependent gene expression was also impaired. Several of these common RNF20/RNF40/VDR targets were shown to be downregulated in experimental colitis, such as $M u c 2$ [36]. This finding is in agreement with our recent publication describing that H2Bub1 controls early stages of osteoblast differentiation and bone cell crosstalk by regulating the expression of the VDR target gene Tnfsfl1 [37]. In general, the finding that VDR-dependent genes are frequently downregulated in IBD supports vitamin D supplementation in clinical practice as well as experimental trials. Whether the presence of H2Bub1 may potentially serve as a predictive biomarker for vitamin $\mathrm{D}$ responsiveness in IBD patients remains to be elucidated. However, the observation that neither Rnf20/40-deficient mice, nor patients with low expression of RNF20/40-dependent genes displayed vitamin D deficiency suggests that vitamin D supplementation alone would likely not be sufficient to compensate for decreased vitamin D receptor levels.

Despite the common deregulation of $V d r$ and VDR target genes, our mRNA-seq data demonstrate that some genes are differentially affected by the deletion of Rnf 20 and Rnf 40 in IECs. This finding suggests that RNF20 and RNF40 may have additional, unique ubiquitination targets in addition to $\mathrm{H} 2 \mathrm{~B}$ involved in the regulation of gene expression. Future mass spectrometry-based identification of ubiquitinated proteins in the presence or absence of RNF20/40 is needed to clarify the distinct functions of RNF20 and RNF40.

Interestingly, the role of H2Bub1 in controlling intestinal inflammation appears to be dramatically different depending upon the degree to which it is lost. In a previous study, we employed a different conditional knockout model for the distal intestinal epithelium (CAC-Cre) which results in a low knockout efficiency [38]. Using this model to delete Rnf40 resulted in a sporadic loss of RNF40 and H2Bub1 staining in only $15 \%$ of colorectal epithelial cells and paradoxically elicited a protective effect during DSSinduced colitis. This finding is in agreement with the current study in which the inefficient reduction of Rnf20 or Rnf 40 in heterozygous animals did not promote colorectal inflammation. Notably, when evaluating why heterozygous mice only displayed minor differences when compared to wildtype controls, we detected that H2Bub1 staining intensity was similar across wild-type and heterozygous genotypes. Thus, we hypothesize that only a complete loss of H2Bub1 in the intestinal epithelium is sufficient to elicit spontaneous colitis.

The H2B monoubiquitination pathway likely plays multiple roles in controlling IBD in different cell types. Interestingly, a recent CRISPR screen identified RNF20 as a negative regulator of the master regulatory $\mathrm{T}$ cell (Treg) transcription factor Forkhead box P3 (FOXP3) [5]. In fact, FOXP3-positive Tregs play an important role in IBD [6, 7]. Similar divergent cell type-dependent differential functions of an epigenetic regulator in IBD have been demonstrated for the Polycomb Repressive Complex-2 methyltransferase component EZH2. In this case, while global inhibition of EZH2 activity or myeloid-specific Ezh2 deletion ameliorates experimental intestinal inflammation [39] the loss of 
$E z h 2$ in either the intestinal epithelium [15] or regulatory $\mathrm{T}$ cells [7] results in a spontaneous colitis phenotype. Thus, when considering the H2B monoubiquitination pathway as a potential therapeutic target in IBD, it will be essential to determine and consider the differential roles it plays in diverse cell types important for IBD biology.

In conclusion, this work describes a previously unknown role of RNF20/40-mediated H2B monoubiquitination in the establishment of broad $\mathrm{H} 3 \mathrm{~K} 4 \mathrm{me} 3$ domains, which appear to promote the transcriptional elongation of VDR-dependent genes associated with intestinal inflammation. In vivo experiments revealed that H2Bub1-deficient mice developed spontaneous colorectal inflammation and, in support of these findings, we detected that $80 \%$ of IBD patients displayed a loss of H2Bub1 in inflamed areas. Notably, we detected a subgroup of IBD patients who display deregulated expression and epigenetic control of RNF20/40dependent gene signatures affecting $V D R$ and VDR target genes. Based on this, additional studies will be essential to uncover the interdependence of VDR activity and H2B monoubiquitination and determine the potential therapeutic utility of targeting these for the benefit of patients with IBD.

\section{Methods}

\section{Patient work}

To evaluate H2Bub1 levels in IBD sections, resection specimens of 18 Crohn's disease patients (Supplementary Table S1) were analyzed with approval of the local ethics committee of the University Medical Center Goettingen, Germany (registration number: 1/10/19). For each patient, inflamed epithelium, as well as healthy resection margins, were assessed. Moreover, H3K27ac occupancy was analyzed in resection specimens of 30 Crohn's disease patients using chromatin immunoprecipitation (ChIP) as approved by the local ethics committee of the Mayo Clinic, Rochester, MN, USA (Supplementary Table S2; registration number: IRB \#18-002942). All research was performed in accordance with the principles expressed in the Declaration of Helsinki, all study participants voluntarily participated in the study and gave informed consent.

\section{Animal work}

The $R n f 20^{\text {tmlc(EUCOMM)Wtsi }}$ mouse line, referred to here as $R n f 20^{\text {flox }}$, containing loxP sites flanking exons 3-5 was generated from the mouse embryonic stem cell line G11 (EPD0701_3_G11) purchased from the European Conditional Mouse Mutagenesis Program (EUCOMM) by crossing "knockout first" allele (Rnf2 $\left.0^{\mathrm{tmla}(\mathrm{EUCOMM}) \mathrm{Wtsi}}\right)$ mice with a mouse line ubiquitously expressing the Flp recombinase (C57BL/6 N(Cag-FlpO)1Afst/Mmcd) [40]. The $R n f 40^{\text {flox }}$ mouse line with loxP sites flanking exons 3-4 was described previously[12]. Both $R n f 20^{\text {flox }}$ and $R n f 40^{\text {flox }}$ lines were crossed with Villin-CreER ${ }^{\mathrm{T} 2}$ [41] in a C57BL/6 $\mathrm{N}$ background to achieve tamoxifen-inducible intestinal deletion of floxed Rnf20 or Rnf40 alleles. For knockout induction, mice were intraperitoneally injected with a dose of $1 \mathrm{mg}$ tamoxifen (Sigma-Aldrich Co., St. Louis, USA) per day for 5 consecutive days at the age of 6 weeks. Numbers of mice sacrificed 14 days after the first tamoxifen injection were $n=10\left(\operatorname{Rnf} 20 / 40^{\mathrm{wt} / \mathrm{wt}}\right), n=7\left(R n f 20^{\mathrm{wt} / \mathrm{fl}}\right)$, $n=8\left(\operatorname{Rnf} 20^{\mathrm{f} / \mathrm{fl}}\right), n=7\left(\operatorname{Rnf} 40^{\mathrm{wt} / \mathrm{fl}}\right), n=17\left(\operatorname{Rnf} 40^{\mathrm{t} / \mathrm{fl}}\right)$ and mice observed over a period of 6 months $n=8\left(\right.$ Rnf20/40 $\left.0^{\mathrm{wt} / \mathrm{wt}}\right)$, $n=10 \quad\left(R n f 2 O^{\mathrm{wt} / \mathrm{fl}}\right), \quad n=7 \quad\left(\operatorname{Rnf} 40^{\mathrm{w} / \mathrm{fl}}\right)$. To induce acute colitis, animals were treated with $0.75 \%$ dextran sodium sulfate (DSS; MO Biomedicals, LLC, Illkirch, France) for 14 consecutive days as previously described [3] $(n=9$ $\left.\left(\operatorname{Rnf} 20 / 40^{\mathrm{wt} / \mathrm{wt}}\right), n=10\left(\operatorname{Rnf} 20^{\mathrm{wt} / \mathrm{fl}}\right), n=7\left(\operatorname{Rnf} 40^{\mathrm{wt} / \mathrm{fl}}\right)\right)$.

\section{Mouse genotyping}

DNA was isolated using a Mouse Direct PCR Kit (Stratech Scientific, Ely, UK). Upon an initial denaturation step at $94{ }^{\circ} \mathrm{C}$ for $2 \mathrm{~min}$, DNA fragments were amplified as follows: 34 cycles of $94{ }^{\circ} \mathrm{C}$ for $20 \mathrm{~s}, 58^{\circ} \mathrm{C}$ for $30 \mathrm{~s}$ and $72^{\circ} \mathrm{C}$ for 1 $\min \left(\right.$ Rnf20); 34 cycles of $94{ }^{\circ} \mathrm{C}$ for $20 \mathrm{~s}, 62^{\circ} \mathrm{C}$ for $30 \mathrm{~s}$ and $72{ }^{\circ} \mathrm{C}$ for $1 \mathrm{~min}\left(\right.$ Rnf 40 ); 34 cycles of $94^{\circ} \mathrm{C}$ for $20 \mathrm{~s}, 56^{\circ} \mathrm{C}$ for $30 \mathrm{~s}$ and $72^{\circ} \mathrm{C}$ for $1 \mathrm{~min}$ (Cre-recombinase) with a final elongation at $72{ }^{\circ} \mathrm{C}$ for $5 \mathrm{~min}$. Primer sequences are listed in Supplementary Table S3. Fragments were subsequently analyzed by agarose gel electrophoresis.

\section{Determination of the Disease Activity Index (DAI)}

To monitor health condition of mice, the DAI was calculated as previously described [3]. Briefly, the DAI includes scores for weight loss $(0: 0-1 \% ; 1: 1-5 \% ; 2: 5-10 \%$; 3 : $10-15 \% ; 4:>15 \%)$, stool consistency ( 0 : normal; 1 : soft; 2 : very soft; 3 : diarrhea) and the presence of occult blood as determined using the stool guaiac test ( 0 : no blue staining; 1: weak/sporadic staining; 2: medium staining; 3 : strong blue staining; 4: bloody anus). Investigators were blinded during mouse handling including the determination of the disease activity index.

\section{Isolation of intestinal epithelial cells (IECs)}

Upon sacrificing Villin-CreER ${ }^{\mathrm{T} 2}, R n f 20 / 40^{\mathrm{wt} / \mathrm{wt}}, R n f 20^{\mathrm{f} / \mathrm{fl}}$ and $R n f 40^{\mathrm{f} / \mathrm{fl}}$ mice fourteen days upon the first tamoxifen injection, colons were isolated $(n=3$ pooled/genotype $)$ and flushed with ice-cold PBS. Colons were opened longitudinally and washed in ice-cold PBS. A total of $<1 \mathrm{~mm}^{2}$ fragments were incubated with collagenase type I $\left(\mathrm{GIBCO}^{\circ}\right.$, 
Invitrogen $\mathrm{GmbH}$, Darmstadt, Germany) solution $2 \mathrm{mg} / \mathrm{ml}$ collagenase in washing medium (DMEM/F12 + HEPES supplemented with $10 \%$ fetal bovine serum (FBS), 100 units $/ \mathrm{ml}$ penicillin, and $100 \mu \mathrm{g} / \mathrm{ml}$ streptomycin) at $37^{\circ} \mathrm{C}$ for approximately $30-40 \mathrm{~min}$. Suspensions were pipetted vigorously up and down until $50-80 \%$ of the crypts were separated from the muscle layer. Next, suspensions were transferred to tubes containing $10 \mathrm{ml}$ washing medium. Upon centrifugation at $250 \mathrm{xg}$ for $5 \mathrm{~min}$, supernatants containing muscle fragments were aspirated and the cell pellets were re-suspended in $10 \mathrm{ml}$ washing medium. For subsequent RNA, ChIP and protein analyses, IEC pellets were snap-frozen in liquid nitrogen and stored at $-150{ }^{\circ} \mathrm{C}$. For cell culture experiments, wild type IECs were resuspended in growth medium.

\section{IEC cell culture and transfections}

IECs were grown in Dulbecco's Modified Eagle Medium (DMEM) supplemented with 20\% FBS, 100 units $/ \mathrm{ml}$ penicillin and $100 \mu \mathrm{g} / \mathrm{ml}$ streptomycin at $37{ }^{\circ} \mathrm{C}$ and $5 \%$ $\mathrm{CO}_{2}$. To transiently deplete RNF20 and RNF40, siRNA (GE Dharmacon siGENOME; non-targeting siRNA 5 [D001210-05-20], RNF20 siRNAs (D-041733-01, -02, -03, -04), RNF40 siRNAs (D-059014-01, -02, -03, -04)) transfections were performed using Lipofectamine ${ }^{\circ}$ RAiMAX (Invitrogen GmbH, Karlsruhe, Germany) according to the manufacturer's instructions. After $24 \mathrm{~h}$, cells were treated with $1 \mu \mathrm{M}$ calcitriol (Biomol, Hamburg, Germany), the active form of vitamin D, dissolved in DMSO or DMSO alone for $48 \mathrm{~h}$. For $V D R$ overexpression, IECs were transfected with a VDR-eGFP expression plasmid [42] or a control plasmid using Lipofectamine 2000 (Invitrogen $\mathrm{GmbH}$, Karlsruhe, Germany) according to the manufacturer's instructions. Plasmids were a kind gift from Rajiv Kumar and Theodore A. Craig (Department of Internal Medicine, Division of Nephrology and Hypertension, Mayo Clinic, Rochester, MN). Forty-eight hours after transfection, siRNAs were added and 72 hours after knockdown, RNA was isolated. Overexpression of $V D R$ was verified by qRTPCR and subsequent agarose gel electrophoresis.

\section{Histology and determination of the Histo-score (H- score)}

Hematoxylin and Eosin (H\&E) staining and immunohistochemical (IHC) staining of paraffin sections were performed as previously described [3, 43]. Antibodies utilized for IHC are listed in Supplementary Table S4. To determine inflammation intensity, the $\mathrm{H}$-score was determined on H\&E-stained colon sections as previously described [3]. It ranges from 0 to 3 , considering normal epithelium (0), as well as intestinal areas displaying mild [1], medium [2], or severe [3] epithelial damage. Investigators were blinded during the analysis of the $\mathrm{H}$-score and IHC stainings.

\section{Western blotting and quantitative real-time PCR (qRT-PCR)}

Protein isolation, western blot analysis, RNA extraction, reverse transcription, and qRT-PCR were performed as previously described [17]. Gene expression levels were assessed and normalized to the housekeeping gene Rplp0. Primary antibodies for western blot and primers for qRT-PCR are listed in Supplementary Tables S4 and S5, respectively.

\section{mRNA-seq and analysis}

mRNA sequencing libraries were prepared using the TruSeq RNA Library Prep V2 kit (Illumina ${ }^{\circ}$ ) and sequencing was performed on a HiSeq4000 (Illumina) at the Genome Analysis Core, Mayo Clinic as reported earlier [43]. Fastq files were mapped to the mouse reference genome $\mathrm{mm} 9$ and analysis was performed as previously described [43]. All data have been deposited at ArrayExpress (http://www.ebi. ac.uk/arrayexpress, accession number: E-MTAB-9405). Gene set enrichment analysis (GSEA) [44, 45] was performed using custom gene sets containing genes significantly regulated in mice undergoing acute DSS-induced colitis [23] and in inflamed intestinal biopsies isolated from patients displaying severe IBD [24]. In addition, genes differentially regulated in small intestines (SI) of vitamin Dtreated mice [29] were compared to our own data.

\section{Chromatin immunoprecipitation (ChIP) and ChIP- seq}

Snap-frozen IECs were thawed on ice, resuspended in $8 \mathrm{ml}$ of $1 \%$ formaldehyde in PBS and incubated at room temperature on a rolling device for $20 \mathrm{~min}$. ChIP was performed as previously described [17].

Chromatin preparation from formalin-fixed paraffinembedded (FFPE) resection specimens from 30 Crohn's disease patients was performed following the published ChromEX-PE technology [46] with slight modifications. Briefly, two $20 \mu \mathrm{m}$ sections were deparaffinized and rehydrated. Tissues were incubated in $500 \mu \mathrm{l}$ of chromatin stabilization buffer $(10 \mathrm{mM}$ Tris- $\mathrm{HCl}, \mathrm{pH} 7.5,10 \mathrm{mM} \mathrm{NaCl}$, $10 \mathrm{mM}$ EDTA, $0.5 \%$ Triton $\mathrm{X}-100,0.1 \%$ sodium deoxycholate, $20 \% \mathrm{EtOH}$ ) at $65^{\circ} \mathrm{C}$ overnight and centrifuged at $21,130 \mathrm{xg}$ at $4{ }^{\circ} \mathrm{C}$ for $5 \mathrm{~min}$. Tissue pellets were resuspended in $250 \mu \mathrm{l}$ RIPA buffer $(10 \mathrm{mM}$ Tris- $\mathrm{HCl}, \mathrm{pH} 8.0$, $140 \mathrm{mM} \mathrm{NaCl}, 1 \mathrm{mM}$ EDTA, $1 \%$ Triton X-100, 0.1\% SDS) and lysates were sonicated ( 20 cycles, $30 \mathrm{sec}$ on/off) using a Bioruptor Pico sonicator (Diagenode, Inc., Denville, NJ). Supernatants (chromatin inputs) were collected after 
centrifugation $\left(21,130 \mathrm{xg}\right.$ at $4{ }^{\circ} \mathrm{C}$ for $\left.5 \mathrm{~min}\right)$ and were incubated with $0.2 \mu \mathrm{g}$ anti-H3K27ac antibody overnight. After adding $15 \mu \mathrm{l}$ of protein G-magnetic beads, the reactions were further incubated for 3 hours. The beads were extensively washed with ChIP buffer, high salt buffer, $\mathrm{LiCl}_{2}$ buffer, and TE buffer. Bound chromatin was eluted and reverse-crosslinked at $65^{\circ} \mathrm{C}$ overnight. DNA was purified using the MinElute PCR purification kit (Qiagen, Valencia, CA) after treatment with RNase $\mathrm{A}$ and proteinase $\mathrm{K}$. Antibody information is listed in Supplementary Table S4 and ChIP-qPCR primers in Supplementary Table S6.

IEC ChIP libraries were prepared using the KAPA Hyper Prep Kit (KAPABiosystems, Cape Town, South Africa) and H3K27ac libraries (patient samples) using the ThruPLEX ${ }^{\oplus}$ DNA-seq Kit V2 (Rubicon Genomics, Ann Arbor, MI, USA). Library quality was estimated using an Agilent Bioanalyzer 2100 and samples were sequenced (single-end 50 bp) on a HiSeq4000 (Illumina , Genome Analysis Core, Mayo Clinic). Reads were mapped to the murine reference genome $\mathrm{mm} 9$ and analysis was performed as previously described [17]. Occupancy profiles were viewed using Integrative Genomics Viewer (IGV 2.5.0) [29]. In addition, to evaluate VDR occupancy, previously published ChIP-seq data [28] have been compared to our own sequencing results. H3K27ac libraries were prepared using the ThruPLEX ${ }^{\oplus}$ DNA-seq Kit V2 (Rubicon Genomics, Ann Arbor, MI, USA) and sequenced to 51 base pairs from both ends on a HiSeq4000 (Illumina ${ }^{\circ}$ ) in the Mayo Clinic Center for Individualized Medicine Medical Genomics Facility. The enrichment was analyzed by targeted real-time PCR in positive and negative genomic loci. H3K27ac ChIP-seq data were processed using the internal HiChIP pipeline to generate library-size normalized signal tracks for visualization and a list of peaks [30]. Briefly, paired-end reads were mapped to the human reference genome (hg38) by Burrows-Wheeler Alignment [31] with default settings and only pairs with at least one of the ends being uniquely mapped were retained for further analysis. Alignments were position sorted and duplicates were removed using Picard tools (https://broadinstitute.github.io/picard/). Peaks were called using the MACS2 algorithm at FDR $\leq 1 \%$. Differential binding peaks for patients with RNF20/40-associated gene signatures were called using the DiffBind $\mathrm{R}$ package [32]. Peaks with $p$ value $<1 \mathrm{e}-5$ and absolute $\log 2$ fold change $>1$ were retained as significant. ChIP-seq data have been deposited at ArrayExpress (http://www.ebi.ac.uk/arra yexpress, accession E-MTAB-9409).

\section{Single cell RNA-seq (scRNA-seq)}

Publically available single cell RNA-seq data based on uninflamed and inflamed intestinal resection specimens isolated from Crohn's disease patients $(n=11)$ were analyzed as previously described [20]. Briefly, sequencing data were aligned to the human reference genome Grch38, and data with at least 500 unique molecular identifiers (UMIs), $\log 10$ genes per UMI $>0.8,>250$ genes per cell and a mitochondrial ratio of less than $0.2 \%$ were extracted, normalized and integrated using the seurat package v3.0 in $\mathrm{R}$ 4.0.2.

\section{Statistics}

Graphs were generated with GraphPad Prism version 8.0.1 (GraphPad Software, Inc.), R version 3.6.2 or 4.0.2 and Partek $^{\oplus}$ Genomics Suite ${ }^{\oplus}$ software version 7.0 (Copyright $^{\circledR}$ 2018; Partek Inc., St. Louis, MO, USA). Categorical variables were represented as $n$, and Chi-square test was used for inter-group comparisons in IBD patient cohorts. Statistical analyses were performed using one-way ANOVA and subsequent Tukey Post hoc test $\left(\alpha=0.05, * p \leq 0.05,{ }^{* *} p \leq\right.$ $0.01, * * * p \leq 0.001)$.

\section{Data availability}

Transcript Profiling Sequencing data have been deposited at ArrayExpress (http://www.ebi.ac.uk/arrayexpress, accession numbers: E-MTAB-9405, E-MTAB-9409).

Author contributions Conceptualization: SAJ, RLK, WAF; Experiments: RLK, MZ, APK, DS, ACS, NP; Bioinformatics: RLK, SAJ, APK, AN, ZY, ZS, Animal experiments: RLK, MZ; Patient work: MvonH, JJF, SZS, MG, RBS, RDN, WAF; Resources: SAJ, MvonH, JJF, SZS, MG, RBS, RDN, WAF; Writing-Original Draft: SAJ and RLK, WAF; Writing-Review and editing: SAJ, RLK, MZ, APK, AN, ZY, DS, MvonH, JJF, ACS, NP, SZS, FW, ZS, MG, RBS, RDN, WAF.

Funding RLK is supported by a Mildred Scheel postdoc fellowship (German Cancer Aid), MZ by the Else Kroener Fresenius Foundation and DS by the German Research Foundation (DFG, 413501650). This study was supported in part by the Helmsley Charitable Trust. Open Access funding enabled and organized by Projekt DEAL.

\section{Compliance with ethical standards}

Ethics statement All animal work was performed according to institutional regulations for the care and use of laboratory animals, and approved by Lower Saxony State Office for Consumer Protection and Food Safety, Germany (registration number: 15/2039). Immunohistochemical studies with primary patient material were analyzed with approval of the local ethics committee of the University Medical Center Goettingen, Germany (registration number: 1/10/19). Analysis of IBD patient samples by ChIP-seq was approved by the ethics committee of the Mayo Clinic, Rochester, MN, USA (registration number: IRB \#18- 002942).

Conflict of interest The authors declare no competing interests.

Publisher's note Springer Nature remains neutral with regard to jurisdictional claims in published maps and institutional affiliations. 
Open Access This article is licensed under a Creative Commons Attribution 4.0 International License, which permits use, sharing, adaptation, distribution and reproduction in any medium or format, as long as you give appropriate credit to the original author(s) and the source, provide a link to the Creative Commons license, and indicate if changes were made. The images or other third party material in this article are included in the article's Creative Commons license, unless indicated otherwise in a credit line to the material. If material is not included in the article's Creative Commons license and your intended use is not permitted by statutory regulation or exceeds the permitted use, you will need to obtain permission directly from the copyright holder. To view a copy of this license, visit http://creativecommons. org/licenses/by/4.0/.

\section{References}

1. Molodecky NA, Soon IS, Rabi DM, Ghali WA, Ferris M, Chernoff $\mathrm{G}$, et al. Increasing incidence and prevalence of the inflammatory bowel diseases with time, based on systematic review. Gastroenterology. 2012;142:46-54.

2. Rappaport SM. Redefining environmental exposure for disease etiology. NPJ Syst Biol Appl. 2018;4:30.

3. Kosinsky RL, Chua RL, Qui M, Saul D, Mehlich D, Ströbel P, et al. Loss of RNF40 Decreases NF-אB Activity in Colorectal Cancer Cells and Reduces Colitis Burden in Mice. J Crohns Colitis. 2019;13:362-73.

4. Tarcic O, Pateras IS, Cooks T, Shema E, Kanterman J, Ashkenazi $\mathrm{H}$, et al. RNF20 links histone H2B ubiquitylation with inflammation and inflammation-associated cancer. Cell Rep. 2016;14:1462-76.

5. Cortez JT, Montauti E, Shifrut E, Gatchalian J, Zhang Y, Shaked $\mathrm{O}$, et al. CRISPR screen in regulatory $\mathrm{T}$ cells reveals modulators of Foxp3. Nature. 2020;582:416-20.

6. Ban H, Andoh A, Shioya M, Nishida A, Tsujikawa T, Fujiyama $\mathrm{Y}$. Increased number of FoxP3 $+\mathrm{CD} 4+$ regulatory $\mathrm{T}$ cells in inflammatory bowel disease. Mol Med Rep. 2008;1:647-50.

7. Sarmento OF, Svingen PA, Xiong Y, Sun Z, Bamidele AO, Mathison AJ, et al. The role of the histone methyltransferase enhancer of zeste homolog 2 (EZH2) in the pathobiological mechanisms underlying inflammatory bowel disease (IBD). J Biol Chem. 2017;292:706-22.

8. Zhang F, Yu X. WAC, a functional partner of RNF20/40, regulates histone $\mathrm{H} 2 \mathrm{~B}$ ubiquitination and gene transcription. Mol Cell. 2011;41:384-97.

9. Johnsen SA. The enigmatic role of H2Bub1 in cancer. FEBS Lett. 2012;586:1592-601.

10. Sun Z-W, Allis CD. Ubiquitination of histone $\mathrm{H} 2 \mathrm{~B}$ regulates $\mathrm{H} 3$ methylation and gene silencing in yeast. Nature. 2002;418: 104-8.

11. Dover J, Schneider J, Tawiah-Boateng MA, Wood A, Dean K, Johnston M, et al. Methylation of histone $\mathrm{H} 3$ by COMPASS requires ubiquitination of histone $\mathrm{H} 2 \mathrm{~B}$ by Rad6. J Biol Chem. 2002;277:28368-71.

12. Xie W, Nagarajan S, Baumgart SJ, Kosinsky RL, Najafova Z, Kari V, et al. RNF40 regulates gene expression in an epigenetic context-dependent manner. Genome Biol. 2017;18:32.

13. Chen K, Chen Z, Wu D, Zhang L, Lin X, Su J, et al. Broad $\mathrm{H} 3 \mathrm{~K} 4 \mathrm{me} 3$ is associated with increased transcription elongation and enhancer activity at tumor-suppressor genes. Nat Genet. 2015;47:1149-57.

14. Kelly D, Kotliar M, Woo V, Jagannathan S, Whitt J, Moncivaiz J, et al. Microbiota-sensitive epigenetic signature predicts inflammation in Crohn's disease. JCI Insight. 2018;3:e122104.

15. Liu Y, Peng J, Sun T, Li N, Zhang LE, Ren J, et al. Epithelial EZH2 serves as an epigenetic determinant in experimental colitis by inhibiting TNF $\alpha$-mediated inflammation and apoptosis. Proc Natl Acad Sci USA. 2017;114:E3796-E3805.

16. Melling N, Grimm N, Simon R, Stahl P, Bokemeyer C, Terracciano $\mathrm{L}$, et al. Loss of H2Bub1 expression is linked to poor prognosis in nodal negative colorectal cancers. Pathol Oncol Res. 2016;22:95-102.

17. Schneider D, Chua RL, Molitor N, Hamdan FH, Rettenmeier EM, Prokakis E, et al. The E3 ubiquitin ligase RNF40 suppresses apoptosis in colorectal cancer cells. Clin Epigenetics. 2019;11:98.

18. Tarcic O, Granit RZ, Pateras IS, Masury H, Maly B, Zwang Y, et al. RNF20 and histone H2B ubiquitylation exert opposing effects in Basal-Like versus luminal breast cancer. Cell Death Differ. 2017;24:694-704.

19. Lim W-C, Hanauer SB, Li YC. Mechanisms of disease: vitamin D and inflammatory bowel disease. Nat Clin $\operatorname{Pr}$ Gastroenterol Hepatol. 2005;2:308-15.

20. Martin JC, Chang C, Boschetti G, Ungaro R, Giri M, Grout JA, et al. Single-cell analysis of crohn's disease lesions identifies a pathogenic cellular module associated with resistance to Anti-TNF therapy. Cell. 2019;178:1493-.e20.

21. Kuleshov MV, Jones MR, Rouillard AD, Fernandez NF, Duan Q, Wang Z, et al. Enrichr: a comprehensive gene set enrichment analysis web server 2016 update. Nucleic Acids Res. 2016;44: W90-7.

22. Chen EY, Tan CM, Kou Y, Duan Q, Wang Z, Meirelles GV, et al. Enrichr: interactive and collaborative HTML5 gene list enrichment analysis tool. BMC Bioinforma. 2013;14:128.

23. Czarnewski P, Parigi SM, Sorini C, Diaz OE, Das S, Gagliani N, et al. Conserved transcriptomic profile between mouse and human colitis allows unsupervised patient stratification. Nat Commun. 2019;10:2892.

24. Haberman Y, Karns R, Dexheimer PJ, Schirmer M, Somekh J, Jurickova I, et al. Ulcerative colitis mucosal transcriptomes reveal mitochondriopathy and personalized mechanisms underlying disease severity and treatment response. Nat Commun. 2019;10:38.

25. Minsky N, Shema E, Field Y, Schuster M, Segal E, Oren M. Monoubiquitinated $\mathrm{H} 2 \mathrm{~B}$ is associated with the transcribed region of highly expressed genes in human cells. Nat Cell Biol. 2008;10:483-8.

26. Pavri R, Zhu B, Li G, Trojer P, Mandal S, Shilatifard A, et al. Histone $\mathrm{H} 2 \mathrm{~B}$ monoubiquitination functions cooperatively with FACT to regulate elongation by RNA polymerase II. Cell. 2006;125:703-17.

27. Visschedijk MC, Alberts R, Mucha S, Deelen P, Jong DJ, de, Pierik M, et al. Pooled resequencing of 122 ulcerative colitis genes in a large dutch cohort suggests population-specific associations of rare variants in MUC2. PLoS One. 2016;11:e0159609.

28. McGovern DPB, Kugathasan S, Cho JH. Genetics of inflammatory bowel diseases. Gastroenterology. 2015;149:1163-76.

29. Lee SM, Riley EM, Meyer MB, Benkusky NA, Plum LA, DeLuca HF, et al. 1,25-Dihydroxyvitamin D3 controls a cohort of vitamin $\mathrm{D}$ receptor target genes in the proximal intestine that is enriched for calcium-regulating components. J Biol Chem. 2015;290: $18199-215$.

30. Ananthakrishnan AN, Khalili H, Higuchi LM, Bao Y, Korzenik JR, Giovannucci EL, et al. Higher predicted vitamin D status is associated with reduced risk of Crohn's disease. Gastroenterology. 2012;142:482-9.

31. Cantorna MT, Munsick C, Bemiss C, Mahon BD. 1,25-Dihydroxycholecalciferol prevents and ameliorates symptoms of experimental murine inflammatory bowel disease. J Nutr. 2000;130:2648-52.

32. Haussler MR, Whitfield GK, Haussler CA, Hsieh JC, Thompson $\mathrm{PD}$, Selznick SH, et al. The nuclear vitamin $\mathrm{D}$ receptor: biological and molecular regulatory properties revealed. J Bone Min Res. 1998;13:325-49. 
33. Simmons JD, Mullighan C, Welsh KI, Jewell DP. Vitamin D receptor gene polymorphism: association with Crohn's disease susceptibility. Gut. 2000;47:211-4.

34. Pei FH, Wang YJ, Gao SL, Liu BR, DU YJ, Liu W, et al. Vitamin D receptor gene polymorphism and ulcerative colitis susceptibility in Han Chinese. J Dig Dis. 2011;12:90-8.

35. Liu W, Chen Y, Golan MA, Annunziata ML, Du J, Dougherty U, et al. Intestinal epithelial vitamin D receptor signaling inhibits experimental colitis. J Clin Invest. 2013;123:3983-96.

36. van der Sluis M, Koning BAEde, Bruijn ACJMde, Velcich A, Meijerink JPP, van Goudoever JB, et al. Muc2-deficient mice spontaneously develop colitis, indicating that MUC2 is critical for colonic protection. Gastroenterology. 2006;131:117-29.

37. Najafova Z, Liu P, Wegwitz F, Ahmad M, Tamon L, Kosinsky $\mathrm{RL}$, et al. RNF40 exerts stage-dependent functions in differentiating osteoblasts and is essential for bone cell crosstalk. Cell Death Differ. 2021;28:700-14.

38. Xue Y, Johnson R, Desmet M, Snyder PW, Fleet JC. Generation of a transgenic mouse for colorectal cancer research with intestinal cre expression limited to the large intestine. Mol Cancer Res. 2010;8:1095-104.

39. Zhou J, Huang S, Wang Z, Huang J, Xu L, Tang X, et al. Targeting EZH2 histone methyltransferase activity alleviates experimental intestinal inflammation. Nat Commun. 2019;10:2427.
40. Kranz A, Fu J, Duerschke K, Weidlich S, Naumann R, Stewart $\mathrm{AF}$, et al. An improved Flp deleter mouse in C57B1/6 based on Flpo recombinase. Genesis. 2010;48:512-20.

41. el Marjou F, Janssen K-P, Chang BH-J, Li M, Hindie V, Chan L, et al. Tissue-specific and inducible Cre-mediated recombination in the gut epithelium. Genesis. 2004;39:186-93.

42. Ryan ZC, Craig TA, Folmes CD, Wang X, Lanza IR, Schaible NS, et al. 1 $\alpha, 25$-Dihydroxyvitamin D3 Regulates Mitochondrial Oxygen Consumption and Dynamics in Human Skeletal Muscle Cells. J Biol Chem. 2016;291:1514-28.

43. Kosinsky RL, Zerche M, Saul D, Wang X, Wohn L, Wegwitz F, et al. USP22 exerts tumor-suppressive functions in colorectal cancer by decreasing mTOR activity. Cell Death Differ. 2020;27:1328-40.

44. Mootha VK, Lindgren CM, Eriksson K-F, Subramanian A, Sihag $\mathrm{S}$, Lehar J, et al. PGC-1alpha-responsive genes involved in oxidative phosphorylation are coordinately downregulated in human diabetes. Nat Genet. 2003;34:267-73.

45. Subramanian A, Tamayo P, Mootha VK, Mukherjee S, Ebert BL, Gillette MA, et al. Gene set enrichment analysis: a knowledgebased approach for interpreting genome-wide expression profiles. Proc Natl Acad Sci USA. 2005;102:15545-50.

46. Zhong J, Ye Z, Clark CR, Lenz SW, Nguyen JH, Yan H, et al. Enhanced and controlled chromatin extraction from FFPE tissues and the application to ChIP-seq. BMC Genomics. 2019;20:249.

\section{Affiliations}

\section{Robyn Laura Kosinsky (iD ${ }^{1} \cdot$ Maria Zerche ${ }^{2} \cdot$ Ana Patricia Kutschat ${ }^{2} \cdot$ Asha Nair $^{3} \cdot$ Zhenqing Ye $^{3} \cdot$ Dominik Saul $^{4}$. Maximilian von Heesen ${ }^{2}$. Jessica J. Friton ${ }^{1}$. Ana Carolina Schwarzer ${ }^{2} \cdot$ Nadia Paglilla $^{2} \cdot$ Shehzad Z. Sheikh $^{5}$. Florian Wegwitz $\mathbb{D}^{2} \cdot$ Zhifu Sun $\mathbb{D}^{3} \cdot$ Michael Ghadimi ${ }^{2} \cdot$ Rodney D. Newberry ${ }^{6} \cdot$ R. Balfour Sartor ${ }^{5}$. William A. Faubion ${ }^{1} \cdot$ Steven A. Johnsen $\mathbb{1}^{2,7}$}

1 Division of Gastroenterology and Hepatology, Mayo Clinic, Rochester, MN, USA

2 Department of General, Visceral and Pediatric Surgery, University Medical Center Goettingen, Goettingen, Germany

3 Division of Biomedical Statistics and Informatics, Mayo Clinic, Rochester, MN, USA

4 Kogod Center on Aging and Division of Endocrinology, Mayo Clinic, Rochester, MN, USA
5 Center for Gastrointestinal Biology and Disease, University of North Carolina at Chapel Hill, Chapel Hill, NC, USA

6 Division of Gastroenterology, Washington University School of Medicine, St. Louis, MO, USA

7 Gene Regulatory Mechanisms and Molecular Epigenetics Laboratory, Division of Gastroenterology and Hepatology, Mayo Clinic, Rochester, MN, USA 